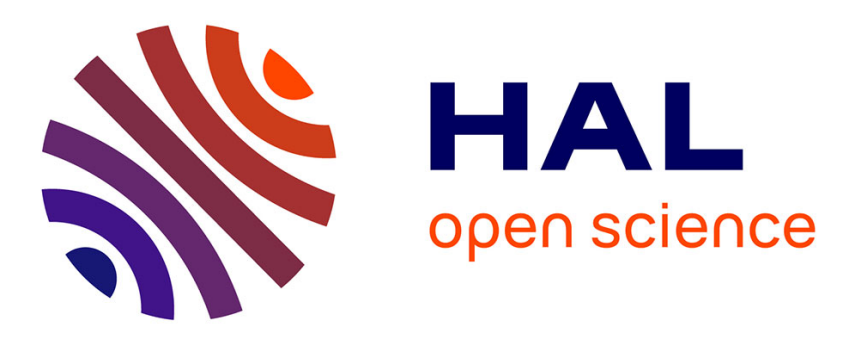

\title{
Memfractance: A Mathematical Paradigm for Circuit Elements with Memory
}

\author{
Mohammed-Salah Abdelouahab, René Lozi, Leon L. O. Chua
}

\section{To cite this version:}

Mohammed-Salah Abdelouahab, René Lozi, Leon L. O. Chua. Memfractance: A Mathematical Paradigm for Circuit Elements with Memory. International journal of bifurcation and chaos in applied sciences and engineering , 2014, 24 (9), pp.1430023- 1430023-29. 10.1142/S0218127414300237. hal-01322396

\section{HAL Id: hal-01322396 https://hal.science/hal-01322396}

Submitted on 27 May 2016

HAL is a multi-disciplinary open access archive for the deposit and dissemination of scientific research documents, whether they are published or not. The documents may come from teaching and research institutions in France or abroad, or from public or private research centers.
L'archive ouverte pluridisciplinaire HAL, est destinée au dépôt et à la diffusion de documents scientifiques de niveau recherche, publiés ou non, émanant des établissements d'enseignement et de recherche français ou étrangers, des laboratoires publics ou privés. 
Published in

International Journal of Bifurcation and Chaos,

Vol. 24 , No. 9 (2014) pp. $1430023-1$ to $1430023-29$

personnal file

DOI: $10.1142 / S 0218127414300237$

\title{
Memfractance: A Mathematical Paradigm for Circuit Elements with Memory
}

\author{
Mohammed-Salah Abdelouahab \\ Department of Mathematics and Computer Sciences, \\ University Center of Mila, Mila 43000, Algeria \\ medsalah3@yahoo.fr \\ René Lozi \\ Laboratory of Mathematics J. A. Dieudonné, \\ U.M.R. CNRS 7351, University of Nice-Sophia Antipolis, \\ Parc Valrose, 06108 Nice Cedex 02, France \\ r.lozi@unice.fr \\ Leon Chua \\ Department of Electrical Engineering and Computer Sciences, \\ University of California, Berkeley, CA 94720, USA \\ chua@eecs.berkeley.edu
}

Received April 17, 2014

\begin{abstract}
Memristor, the missing fourth passive circuit element predicted forty years ago by Chua was recognized as a nanoscale device in 2008 by researchers of a H. P. Laboratory. Recently the notion of memristive systems was extended to capacitive and inductive elements, namely, memcapacitor and meminductor whose properties depend on the state and history of the system. In this paper, we use fractional calculus to generalize and provide a mathematical paradigm for describing the behavior of such elements with memory. In this framework, we extend Ohm's law to the generalized Ohm's law and prove it.
\end{abstract}

Keywords: Fractance; memfractance; memristor; memcapacitor; meminductor; Ohm's law; fractional calculus.

\section{Introduction}

In his paper, Chua [1971] had predicted the existence of the missing fourth passive circuit element, which is characterized by a constitutive nonlinear relationship between the charge $q$ and the flux $\varphi$. Such a physical device would not be reported until 2008, when a physical model of a two-terminal hp device behaving as a memristor was announced in [Strukov et al., 2008]. This element shows many interesting features when describing electrical phenomena, especially at small (molecular or cellular) scales. In fact, the memristor is a device that does not depend on any particular material or physical mechanism. For example, spin-transfer magnetic tunnel junctions [Chua, 2011] are also memristors even though the physical memristive mechanism is completely different from the hp memristor. A memristor is also useful for bioimpedance in bioelectricity modeling [Johnsen, 2012].

In [Gale et al., 2014] a simpler method to make memristors for testing purpose has been demonstrated. These drop-coated $\mathrm{Al} / \mathrm{TiO}_{2} / \mathrm{Al}$ memristors undergo memristive Bi-Polar Switching. These devices can be synthesized with equipment available 
in a standard chemistry lab, simplifying the methodology still further and widening the field of researchers who can experiment with memristors.

Recently [Di Ventra et al., 2009], the notion of memristive systems was extended to capacitive and inductive elements, namely, capacitors and inductors whose properties depend on the state and history of the system. The authors argue that these devices are common at the nanoscale, where the dynamical properties of electrons and ions are likely to depend on the history of the system, at least within certain time scales. These elements (memristor, memcapacitor, and meminductor) and their combination in circuits open up new functionalities in electronics and are likely to find applications in neuromorphic devices to stimulate learning, adaptative, and spontaneous behavior. Fractional calculus [Butzer \& Westphal, 2000] which is a generalization of integration and differentiation to arbitrary order, allows us to generalize equations of classic electric elements (resistor, capacitor and inductor) to fractance elements [Le MeHaute \& Crepy, 1983]. In this paper, we use fractional calculus in order to generalize and provide a mathematical paradigm for circuit elements with memory: the memfractance. In this framework, we extend Ohm's law to the generalized Ohm's law for memory elements and prove it. The motivation and signification of this paper is that there may exist future nanoelectronics devices that are more realistically modeled with memfractance elements. Several illustrative examples are explored theoretically and numerically, using the new tool of interpolated memfractance of four memory elements: memristor, memcapacitor, meminductor and the newly introduced secondorder memristor, respectively. A special attention is devoted to the interpolated characteristic of a memfractor lying between memristor and memcapacitor which exhibits an unexpected new behavior of time variation of flux ( $\varphi-t$ curve). This phenomenon is studied very carefully by the means of rigorous proofs. Finally, following Chua who has introduced the notion of $(\alpha, \beta)$ elements which defines an infinite discrete family of circuit elements, which is essential for developing a rigorous mathematical theory of nonlinear circuits, we extend the generalized Ohm's law in order to embed memfractor elements into this periodic table. In this scope, we define an infinite continued family of circuit elements with a special metric. We call it - fractional circuit element family. In Sec. 2, we recall the general background of both circuit elements with memory and fractional calculus. We generalize the definition of fractance and we give the related Ohm's law. In Sec. 3, the second-order memristor is defined, the paradigm of memfractance is introduced and the generalized Ohm's law for memory elements is proved. In Sec. 4, the interpolated memfractance is carefully studied in order to consider particular memfractor elements. Numerical illustrative examples are computed and their results displayed in Sec. 5. A special attention is devoted to the interpolated characteristic of a memfractor lying between memristor and memcapacitor. Finally, in Sec. 6, we extend the generalized Ohm's law to the periodic table of circuit elements.

\section{General Backgrounds}

In this section, we recall some preliminary concepts of circuit elements with memory, fractional calculus and fractance element.

\subsection{Circuit elements with memory}

The general class of $n$ th-order $u$-controlled memory devices are defined [Chua \& Kang, 1976; Chua, 2003, 2009, 2012] as those described by the following relations:

$$
\left\{\begin{array}{l}
y(t)=g(x, u, t) u(t), \\
\dot{x}=f(x, u, t)
\end{array}\right.
$$

where $f$ is a continuous $n$-dimensional vector function, $x$ denotes a set of $n$ state variables describing the internal state of the system, $u(t)$ and $y(t)$ are any two complementary constitutive variables [Chua, 2003] denoting input and output of the system (i.e. current, charge, voltage or flux) and where $g$ is a generalized response satisfying $|g(x, u, t)|<\infty$ for $|u(t)|<\infty$.

Memcapacitive, memristive and meminductive systems are special cases of (1), where the two constitutive variables that define them are charge and voltage for the memcapacitance, current and voltage for memristance and, current and flux for the meminductance. Following [Chua \& Kang, 1976; Chua, 2012], we give a brief definition of circuit elements with memory.

Definition 2.1. An $n$ th-order current-controlled memristor is defined by

$$
\left\{\begin{array}{l}
v_{R}(t)=R(x, i, t) i(t), \\
\dot{x}=f(x, i, t)
\end{array}\right.
$$


where $v(t)$ and $i(t)$ denote the voltage and current across the device and $R$ is a scalar, called the memristance.

The ideal memristor is a special case of generic memristor when $R$ depends only on charge, namely

$$
v_{R}(t)=R(q(t)) i(t) .
$$

Considering devices with $R(x, i, t)>0$, it has been proved [Chua \& Kang, 1976] that these devices are passive and possess the no energy discharge property.

Definition 2.2. An $n$ th-order charge-controlled memcapacitor is defined by

$$
\left\{\begin{array}{l}
v_{C}(t)=C^{-1}(x, q, t) q(t), \\
\dot{x}=f(x, q, t),
\end{array}\right.
$$

where $C^{-1}$ denotes the inverse memcapacitance. The ideal memcapacitor is a special case of the generic memcapacitor when $C^{-1}$ depends only on the integral of charge, namely

$$
v_{C}(t)=C^{-1}\left(\int_{-\infty}^{t} q(\tau) d \tau\right) q(t) .
$$

Definition 2.3. An $n$ th-order current-controlled meminductor is defined by

$$
\left\{\begin{array}{l}
\varphi(t)=L(x, i, t) i(t), \\
\dot{x}=f(x, i, t)
\end{array}\right.
$$

where $L$ is the meminductance and $\varphi(t)$ is the flux defined by $\varphi(t)=\int_{-\infty}^{t} v_{L}(\tau) d \tau$. The ideal meminductor is a special case of the generic meminductor when $L$ depends only on the integral of current, namely

$$
\varphi_{L}(t)=L\left(\int_{-\infty}^{t} i(\tau) d \tau\right) i(t) .
$$

Remark 2.1. It is important to stress that " $q$ " and " $\varphi$ " are defined mathematically and need not have any physical interpretation.

Figure 1 shows three symbols used for the devices defined above as presented in [Di Ventra et al., 2009; Chua, 2009].

\subsection{Fractional calculus}

The idea of fractional calculus has been known since the development of the regular calculus [Leibniz,

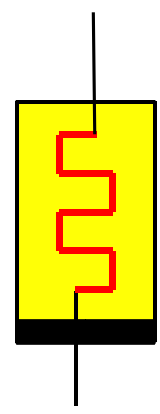

Memristor

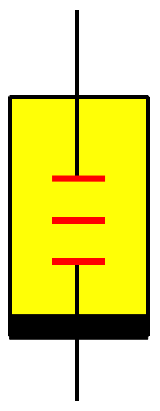

Memcapacitor

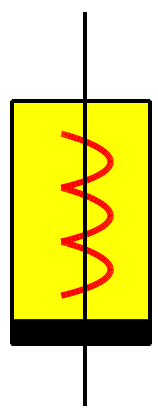

Meminductor
Fig. 1. Symbols of circuit elements with memory (from [Chua, 2009]).

1962] and it means a generalization of integration and differentiation to arbitrary order. It has been found that many systems in interdisciplinary fields can be described by the fractional differential equations, such as viscoelastic systems, dielectric polarization, electrode-electrolyte polarization, electromagnetic waves and quantum evolution of complex systems [Bagley \& Calico, 1991; Sun et al., 1984; Tavazoei, 2010; Szabo \& Abonyi, 1965; Kusnezov et al., 1999]. There are several definitions of fractional derivatives [Caputo, 1967; Podlubny, 1999; Samko et al., 1993; Butzer \& Westphal, 2000]. One of the more common definitions is the Riemann-Liouville definition of fractional derivatives [Podlubny, 1999; Samko et al., 1993], given by

$$
\begin{aligned}
&{ }_{a}^{R} D_{t}^{\alpha} f(t)=\frac{1}{\Gamma(n-\alpha)} \frac{d^{n}}{d t^{n}} \int_{a}^{t}(t-\tau)^{n-\alpha-1} f(\tau) d \tau \\
&= \frac{d^{n}}{d t^{n}}\left({ }_{a} j_{t}^{n-\alpha} f(t)\right), \quad t>a, \\
& n-1 \leq \alpha<n,
\end{aligned}
$$

where $\Gamma$ is the gamma function and ${ }_{a} j_{t}^{\beta}$ is the Riemann-Liouville integral operator defined by:

$$
{ }_{a} j_{t}^{\beta} f(t)=\frac{1}{\Gamma(\beta)} \int_{a}^{t}(t-\tau)^{\beta-1} f(\tau) d \tau .
$$

From Eqs. (2.62), (2.69), (2.70) and (2.133) of [Podlubny, 1999] we say that, for a positive integer $n$ and $\alpha \in \mathbf{R}, \beta \in \mathbf{R}^{-}$and under suitable conditions of $f$ we have

$$
\begin{gathered}
{ }_{a}^{R} D_{t}^{\alpha}\left({ }_{a}^{R} D_{t}^{\beta} f(t)\right)={ }_{a}^{R} D_{t}^{\alpha+\beta} f(t), \\
\frac{d^{n}}{d t^{n}}\left({ }_{a}^{R} D_{t}^{\alpha}(f(t))\right)={ }_{a}^{R} D_{t}^{n+\alpha} f(t) .
\end{gathered}
$$


The Laplace transform of the $\alpha$-order RiemannLiouville differential operator is:

$$
\begin{aligned}
L\left\{{ }_{0}^{R} D_{t}^{\alpha} f(t)\right\}= & s^{\alpha} L\{f(t)\} \\
& \left.-\sum_{k=0}^{n-1} s^{k}{ }_{0}^{R} D_{t}^{\alpha-1-k} f(t)\right]_{t=0} .
\end{aligned}
$$

For zero initial conditions we have

$$
L\left\{{ }_{0} D_{t}^{\alpha} f(t)\right\}=s^{\alpha} L\{f(t)\} .
$$

A numerical method often used for calculation of Riemann-Liouville fractional derivative is based on Grünwald-Letnikov definition given by

$$
\begin{aligned}
{ }_{a}^{G} D_{t}^{\alpha} f(t)= & \lim _{h \rightarrow 0} \frac{1}{h^{\alpha}} \sum_{k=0}^{k=\frac{t-a}{h}}(-1)^{k} \\
& \times \frac{\Gamma(\alpha+1)}{k ! \Gamma(\alpha-k+1)} f(t-k h),
\end{aligned}
$$

where $t>a$ and $\alpha$ is a positive real number.

Its integral form is

$$
{ }_{a}^{G} D_{t}^{-\alpha} f(t)=\lim _{h \rightarrow 0} h^{\alpha} \sum_{k=0}^{k=\frac{t-a}{h}} \frac{\Gamma(\alpha+k)}{k ! \Gamma(\alpha)} f(t-k h) .
$$

When $f$ is of class $C^{m}$, where $m-1 \leq \alpha<m$, then Riemann-Liouville definition and GrünwaldLetnikov definition are equivalent [Podlubny, 1999].

Another definition is the Caputo definition of fractional derivatives [Caputo, 1967] which is given by:

$$
\begin{aligned}
{ }_{a} D_{t}^{\alpha} f(t) & =\frac{1}{\Gamma(n-\alpha)} \int_{a}^{t}(t-\tau)^{n-\alpha-1} f^{(n)}(\tau) d \tau \\
& =j^{n-\alpha}\left(\frac{d^{n}}{d t^{n}} f(t)\right), \quad t>a
\end{aligned}
$$

where $n=[\alpha]$ is the value of $\alpha$ rounded up to the nearest integer. The Laplace transform of the $\alpha$ order Caputo differential operator is:

$$
L\left\{{ }_{0} D_{t}^{\alpha} f(t)\right\}=s^{\alpha} L\{f(t)\}-\sum_{k=0}^{n-1} s^{\alpha-1-k} f^{(k)}(0) .
$$

For zero initial conditions we have

$$
L\left\{{ }_{0} D_{t}^{\alpha} f(t)\right\}=s^{\alpha} L\{f(t)\}
$$

and in this case the three cited definitions of fractional derivatives are equivalent.
Remark 2.2. In this paper, we use indifferently the equivalent notations $D_{t}^{\alpha}={ }_{0}^{R} D_{t}^{\alpha}={ }_{0}^{G} D_{t}^{\alpha}={ }_{0} D_{t}^{\alpha}$.

\subsection{Fractance}

The definition equations of classical electric elements: resistor $(R)$, capacitor $(C)$ and inductor $(L)$ (in which $R, C, L$, are constants) are respectively

$$
\begin{aligned}
v(t) & =R i(t), \\
v(t) & =\frac{1}{C} q(t), \\
\varphi(t) & =L i(t) .
\end{aligned}
$$

Taking into account that

$$
\left\{\begin{array}{l}
q(t)=q\left(t_{0}\right)+\int_{t_{0}}^{t} i(\tau) d \tau, \\
\varphi(t)=\varphi\left(t_{0}\right)+\int_{t_{0}}^{t} v(\tau) d \tau,
\end{array}\right.
$$

then the Eqs. (9)-(11) can be written as follows

$$
\begin{aligned}
& v(t)=R i(t), \\
& v(t)=\frac{1}{C} \int_{t_{0}}^{t} i(\tau) d \tau, \\
& v(t)=L \frac{d}{d t} i(t),
\end{aligned}
$$

assuming $v\left(t_{0}\right)=0$ and $q\left(t_{0}\right)=0$.

Let us introduce the following operators $D_{t}^{n}$, $n=-1,0,1$ :

$$
\begin{aligned}
D_{t}^{1} f(t) & =\frac{d}{d t} f(t), \\
D_{t}^{-1} f(t) & =\int_{-\infty}^{t} f(\tau) d \tau \text { and } \\
D_{t}^{0} f(t) & =f(t) .
\end{aligned}
$$

Using the above notations, Eqs. (9)-(11) can be expressed by only one equation as follows [Chua, 2003, 2012]

$$
v(t)=a_{n} D_{t}^{n} i(t)
$$

where

$$
a_{n}= \begin{cases}\frac{1}{C}, & \text { if } n=-1, \\ R, & \text { if } n=0, \\ L, & \text { if } n=1 .\end{cases}
$$


Applying the Laplace transform to both sides of (13) yields $L(v(t))=a_{n} s^{n} L(i(t))$. It follows that $V(s)=a_{n} s^{n} I(s)$ and the impedance is given by

$$
Z(s)=\frac{V(s)}{I(s)}=a_{n} s^{n},
$$

when $n$ is set equal to an arbitrary real number $\alpha$ between -1 and 1, then Eq. (14) gives the impedance $Z(s)=F^{\alpha} s^{\alpha}$ of a fractional electrical element called a "fractance" device. The term fractance was coined by Le MeHaute \& Crepy [1983] for denoting electrical elements with noninteger order impedance. Noting that Eq. (13) can be obtained by applying the inverse Laplace transform $L^{-1}$ to both sides of Eq. (14), so when $n$ is an arbitrary real number $\alpha$ between -1 and 1, Eq. (13) can be written as

$$
v(t)=a_{\alpha} D_{t}^{\alpha} i(t),
$$

which describes the fractance element. In this case $D_{t}^{\alpha}={ }_{0}^{R} D_{t}^{\alpha}$ is a fractional operator (fractional derivative for $\alpha \geq 0$, and fractional integral for $\alpha<0$ ), for the special cases $\alpha=-1, \alpha=0$ and $\alpha=1$ the fractance becomes a capacitor, a resistor and an inductor, respectively, and the interpolated characteristics between these elements can be obtained via the fractance [Radwan et al., 2008], see Fig. 2.

\subsection{Generalized law of fractance}

Equation (15) can be formulated in a more general form that will be useful in the next section; namely,
Eqs. (9)-(11) can be written as

$$
\begin{gathered}
D_{t}^{0} v(t)=R D_{t}^{0} i(t) \quad \text { or } \quad D_{t}^{1} \varphi(t)=R D_{t}^{1} q(t) \\
D_{t}^{0} v(t)=\frac{1}{C} D_{t}^{-1} i(t) \quad \text { or } \quad D_{t}^{1} \varphi(t)=\frac{1}{C} D_{t}^{0} q(t), \\
D_{t}^{-1} v(t)=L D_{t}^{0} i(t) \quad \text { or } \quad D_{t}^{0} \varphi(t)=L D_{t}^{1} q(t)
\end{gathered}
$$

and these three equations can be represented by a single equation [Coopmans et al., 2009] as follows

$$
D_{t}^{\alpha_{1}-1} v(t)=F^{\alpha_{1}, \alpha_{2}} D_{t}^{\alpha_{2}-1} i(t)
$$

or, as $F^{\alpha_{1}, \alpha_{2}}$ is a constant

$$
v(t)=F^{\alpha_{1}, \alpha_{2}} D_{t}^{\alpha_{2}-\alpha_{1}} i(t),
$$

and

$$
D_{t}^{\alpha_{1}} \varphi(t)=F^{\alpha_{1}, \alpha_{2}} D_{t}^{\alpha_{2}} q(t),
$$

or

$$
v(t)=F^{\alpha_{1}, \alpha_{2}} D_{t}^{\alpha_{2}-\alpha_{1}+1} q(t)
$$

where

$$
F^{\alpha_{1}, \alpha_{2}}= \begin{cases}\frac{1}{C}, & \text { if } \alpha_{1}=1, \quad \alpha_{2}=0 \\ R, & \text { if } \alpha_{1}=\alpha_{2}=1 \\ L, & \text { if } \alpha_{1}=0, \quad \alpha_{2}=1\end{cases}
$$

For $\alpha_{1}=\alpha_{2}=1$ we obtain (16), then (20) describes a resistor, for $\alpha_{1}=1, \alpha_{2}=0$, we obtain (17), then (20) describes a capacitor, for

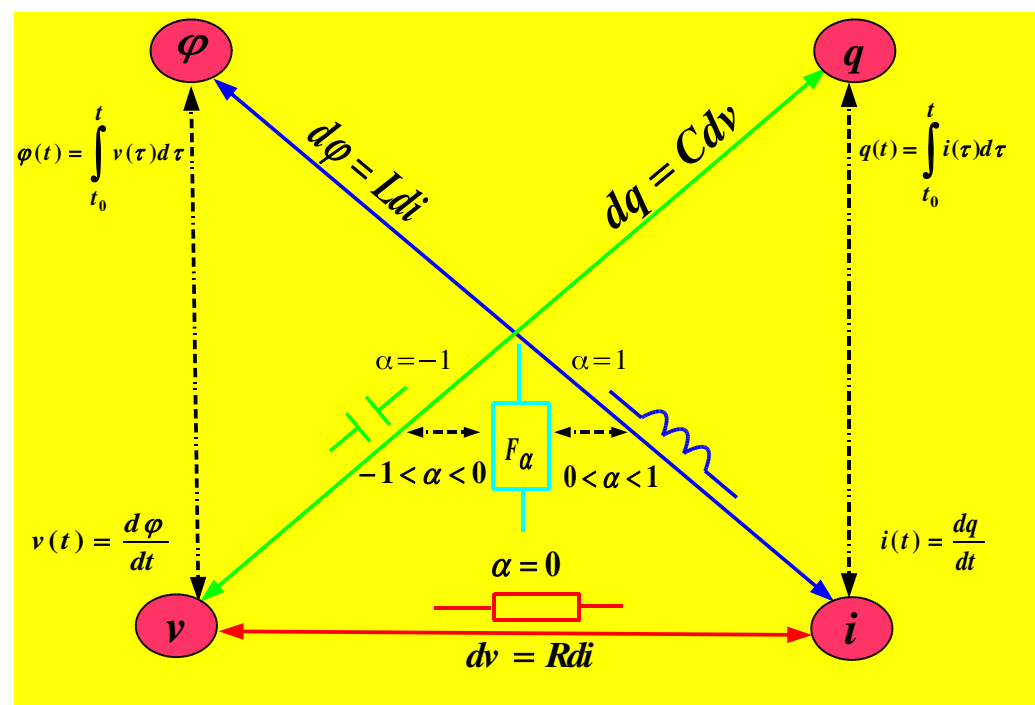

Fig. 2. Interpolated characteristics of fractance between a capacitor, a resistor and an inductor. 
$\alpha_{1}=0, \alpha_{2}=1$, we obtain (18), then (20) describes an inductor.

All the above equations can be generalized so the variables $\alpha_{1}$ and $\alpha_{2}$ are tuned continuously from 0 to 1 as in a homotopy mapping. For $\alpha_{1}, \alpha_{2}$ arbitrary real between 0 and 1, Eq. (20) describes a fractor. The new formulation is more compatible with the representation given in Fig. 2 and will help us in the formulation of the memfractance law. Moreover this two-dimensional definition of fractance extends the previous one-dimensional definition of fractance given by Eq. (15) to a general family of devices when used with elements with memory.

Remark 2.3. If we set $\alpha=\alpha_{2}-\alpha_{1}$ with $-1<\alpha<1$ then Eq. (20) is equivalent to Eq. (15).

\section{Memfractor}

Now let us recall the circuit elements with memory, and we attempt to introduce a fractional memory element which has interpolated characteristics between a memcapacitor, a memristor, a meminductor and a new element called second-order memristor, similarly to that of a fractance element.

\subsection{Second-order memristor}

In this section, we need to introduce a new element which extends the memristor notion (see Definition 2.1) in order to connect the integral of flux $\rho$ to the integral of charge $\sigma$.

Definition 3.1. A second-order charge-controlled memristor is defined by

$$
\left\{\begin{array}{l}
\varphi_{R_{2}}(t)=R_{2}(x, q, t) q(t), \\
\dot{x}=f(x, q, t),
\end{array}\right.
$$

$R_{2}$ is a scalar, called the second-order memristance.

The ideal second-order memristor is a special case of generic second-order memristor when $R_{2}$ depends only on integral of charge $\sigma(t)=$ $\int_{t_{0}}^{t} q(\tau) d \tau$, namely

$$
\varphi_{R_{2}}(t)=R_{2}(\sigma(t)) q(t) .
$$

\subsection{Generalized constitutive relations}

We define the functions $C_{M}^{-1}, R_{M}, L_{M}, R_{2 M}$ and $F_{M}^{\alpha_{1}, \alpha_{2}}$ as follows:

$$
C_{M}^{-1}(t)=C^{-1}(\sigma(t))
$$

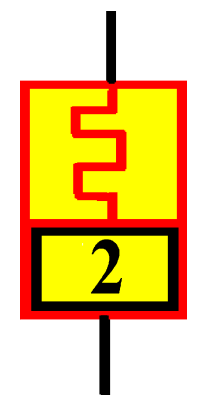

Fig. 3. Symbol of the second-order memristor.

$$
\begin{aligned}
R_{M}(t) & =R(q(t)), \\
L_{M}(t) & =L(q(t)), \\
R_{2 M}(t) & =R_{2}(\sigma(t))
\end{aligned}
$$

and

$$
F_{M}^{\alpha_{1}, \alpha_{2}}(t)= \begin{cases}C_{M}^{-1}(t), & \text { if } \alpha_{1}=1, \quad \alpha_{2}=0, \\ R_{M}(t), & \text { if } \alpha_{1}=\alpha_{2}=1, \\ L_{M}(t), & \text { if } \alpha_{1}=0, \quad \alpha_{2}=1, \\ R_{2 M}(t), & \text { if } \alpha_{1}=0, \quad \alpha_{2}=0\end{cases}
$$

where the lower integration limit (initial moment of time) may be selected as $-\infty$ or zero if $\int_{-\infty}^{0} q(\tau) d \tau=0$. With these notations Eqs. (2)-(4) and (23) can be written as follows

$$
\begin{gathered}
V_{R}(t)=R_{M}(t) I(t), \\
V_{C}(t)=C_{M}^{-1}(t) q(t), \\
\varphi_{L}(t)=L_{M}(t) I(t), \\
\varphi_{R_{2}}(t)=R_{2 M}(t) q(t),
\end{gathered}
$$

which can be written in the form of an operator relationship between flux $\varphi$ and charge $q$ as follows

$$
\begin{aligned}
D_{t}^{1} \varphi_{R}(t) & =R_{M}(t) D_{t}^{1} q(t), \\
D_{t}^{1} \varphi_{C}(t) & =C_{M}^{-1}(t) D_{t}^{0} q(t), \\
D_{t}^{0} \varphi_{L}(t) & =L_{M}(t) D_{t}^{1} q(t), \\
D_{t}^{0} \varphi_{R_{2}}(t) & =R_{2 M}(t) D_{t}^{0} q(t) .
\end{aligned}
$$

Using the fractional operator $D_{t}^{\alpha}$ we introduce a new fractional memory element as follows:

Definition 3.2. We define a memfractor by

$$
D_{t}^{\alpha_{1}} \varphi(t)=F_{M}^{\alpha_{1}, \alpha_{2}}(t) D_{t}^{\alpha_{2}} q(t),
$$

where $\alpha_{1}, \alpha_{2}$ are arbitrary real numbers between 0 and 1 , and $F_{M}^{\alpha_{1}, \alpha_{2}}$ is called memfractance. When 
$\alpha_{1}=1, \alpha_{2}=0$ we obtain a memcapacitor, when $\alpha_{1}=\alpha_{2}=1$ we obtain a memristor, when $\alpha_{1}=0$, $\alpha_{2}=1$ we obtain a meminductor and when $\alpha_{1}=$ $\alpha_{2}=0$ we obtain a second-order memristor. For noninteger values of $\alpha_{1}, \alpha_{2}$ this element possesses interpolated characteristics between a memcapacitor, a memristor, a meminductor and a second-order memristor as depicted in Fig. 4.

When $F_{M}^{\alpha_{1}, \alpha_{2}}(t)=F^{\alpha_{1}, \alpha_{2}}$ is equal to a constant, then (32) describes a fractance element [Eq. (20)].

It follows from this definition that this new postulated element generalizes the classical circuit elements in two ways, first it generalizes them by a continuous variation in the derivative order using the concept of fractional derivative to obtain a fractance element, and secondly, it generalizes them by introduction of memory in its characteristic impedances, to obtain memory circuit elements.

Remark 3.1 (Fractor, Fractductor and Memfractor). Bohannan [2002] introduced a new electrical element called the Fractor, which can be used to emulate the dielectric property of Lithium Hydrazinium Sulfate $\left(\mathrm{LiN}_{2} \mathrm{H}_{5} \mathrm{SO}_{4}\right)$, and exhibits characteristics intermediate between a resistor and a capacitor. In [Coopmans et al., 2009], the authors introduced the fractductor which is intermediate between a resistor and an inductor. More generally, we propose to call

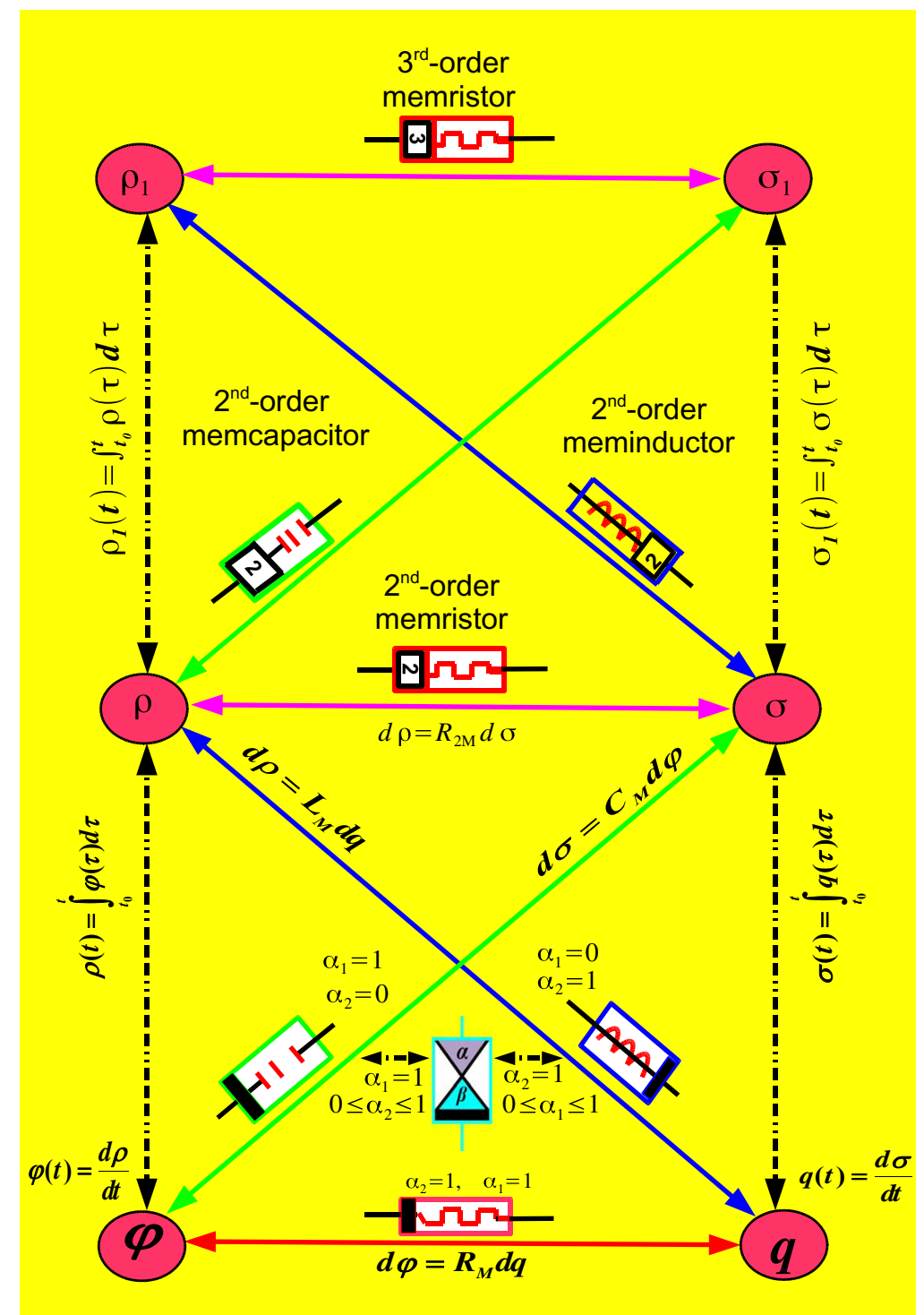

Fig. 4. Interpolated characteristics of memfractor between a memcapacitor, a memristor, a meminductor and a second-order memristor. 
Table 1. Schematic representation of constitutive relationship Eq. (32).

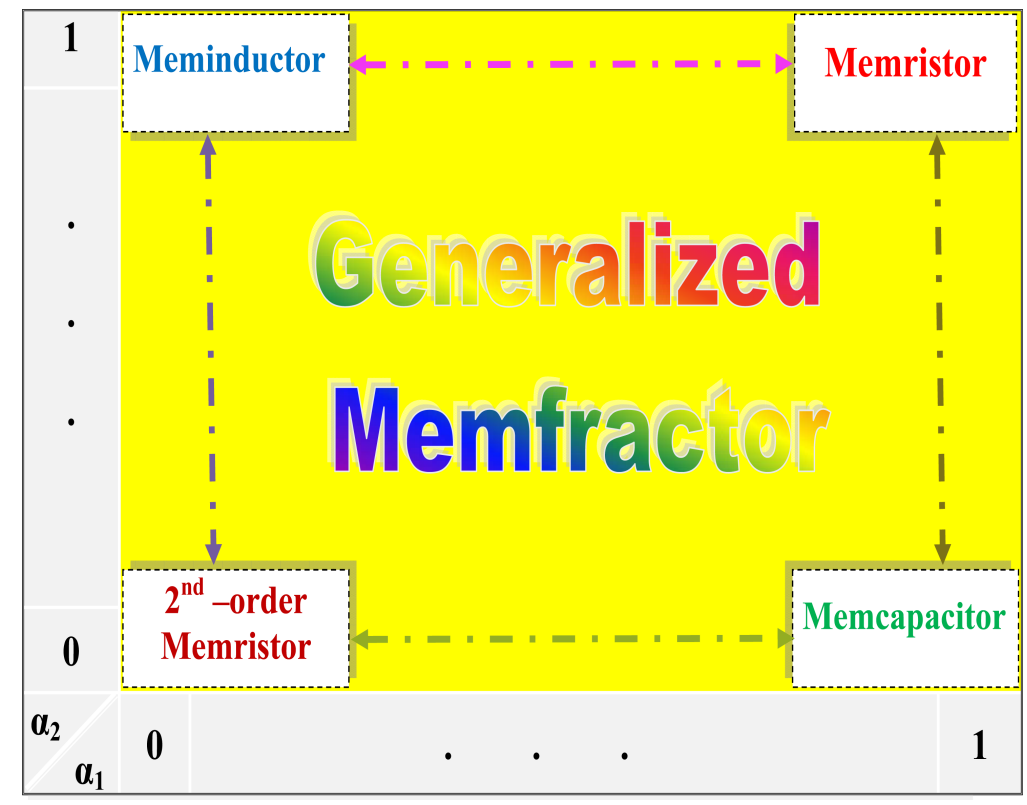

"memfractor" any electrical device which exibits a "memfractance". Memfractor then includes both fractor and fractductor as a particular case.

\subsection{Generalized Ohm's law for memory elements}

The memfractance elements introduced above allow us to generalize Ohm's law for memory elements of electrical circuits as follows:

Proposition 1. The voltage across a memfractance element is given by the relation

$$
v(t)=D_{t}^{1-\alpha_{1}}\left(F_{M}^{\alpha_{1}, \alpha_{2}}(t) D_{t}^{\alpha_{2}} q(t)\right) .
$$

Proof. We have

$$
v(t)=\frac{d \varphi(t)}{d t}=D_{t}^{1} \varphi(t)
$$

thus

$$
\varphi(t)=D_{t}^{-1} v(t)
$$

applying the fractional operator $D_{t}^{\alpha_{1}}$ to both sides of this equation and taking into account relation (5) yields

$$
D_{t}^{\alpha_{1}} \varphi(t)=D_{t}^{\alpha_{1}-1} v(t)
$$

substituting in Eq. (32) we obtain

$$
D_{t}^{\alpha_{1}-1} v(t)=F_{M}^{\alpha_{1}, \alpha_{2}}(t) D_{t}^{\alpha_{2}} q(t) .
$$

Applying the fractional operator $D_{t}^{1-\alpha_{1}}$ to both sides of (35) and taking into account relation (5) leads to Eq. (33), this completes the proof.

Taking into account the relation (5) and the fact that $i(t)=\frac{d}{d t} q(t)$, then the following proposition which gives the relation between the voltage across the memfractance and the current source applied across the memfractance is a straightforward consequence of Proposition 1.

Proposition 2 (Generalized Ohm's Law). The voltage across a memfractance element can be expressed by the relation

$$
v(t)=D_{t}^{1-\alpha_{1}}\left(F_{M}^{\alpha_{1}, \alpha_{2}}(t) D_{t}^{\alpha_{2}-1} i(t)\right) .
$$

Remark 3.2. In this paper, we restrict the domain of validity of Eq. (32) to the values of $\alpha_{1}, \alpha_{2}$ between 0 and 1 as displayed in Table 1.

Remark 3.3. Ohm's law in magnetohydrodynamics is also called generalized Ohm's law [Szabo \& Abonyi, 1965].

\section{Interpolated Memfractance}

The memfractor can be considered in a certain way as an interpolation of the four memory elements (memristor, memcapacitor, meminductor 
Table 2. Values of scalar coefficients.

\begin{tabular}{ccccc}
\hline$\left(\alpha_{1}, \alpha_{2}\right)$ & $a_{\left(\alpha_{1}, \alpha_{2}\right)}$ & $b_{\left(\alpha_{1}, \alpha_{2}\right)}$ & $c_{\left(\alpha_{1}, \alpha_{2}\right)}$ & $d_{\left(\alpha_{1}, \alpha_{2}\right)}$ \\
\hline$(0,0)$ & 0 & 0 & 0 & 1 \\
$(0,1)$ & 0 & 0 & 1 & 0 \\
$(1,0)$ & 1 & 0 & 0 & 0 \\
$(1,1)$ & 0 & 1 & 0 & 0 \\
\hline
\end{tabular}

and second-order memristor). Similarly, the fractional impedance

$$
F_{M}^{\alpha_{1}, \alpha_{2}}(t)=\frac{D_{t}^{\alpha_{1}} \varphi(t)}{D_{t}^{\alpha_{2}} q(t)}, \quad \text { when } D_{t}^{\alpha_{2}} q(t) \neq 0,
$$

can be considered as an interpolation of the memresistance, inverse memcapacitance, meminductance and second-order memresistance, respectively. In this paper, we propose the following form of $F_{M}^{\alpha_{1}, \alpha_{2}}$ as an interpolation of a given memresistance $R_{M}(t)$, inverse memcapacitance $C_{M}^{-1}(t)$, meminductance $L_{M}(t)$ and second-order memresistance $R_{2 M}(t)$; namely,

$$
\begin{aligned}
F_{M}^{\alpha_{1}, \alpha_{2}}(t)= & a_{\left(\alpha_{1}, \alpha_{2}\right)} C_{M}^{-1}(t)+b_{\left(\alpha_{1}, \alpha_{2}\right)} R_{M}(t) \\
& +c_{\left(\alpha_{1}, \alpha_{2}\right)} L_{M}(t)+d_{\left(\alpha_{1}, \alpha_{2}\right)} R_{2 M}(t),
\end{aligned}
$$

where $a_{\left(\alpha_{1}, \alpha_{2}\right)}, b_{\left(\alpha_{1}, \alpha_{2}\right)}, c_{\left(\alpha_{1}, \alpha_{2}\right)}$ and $d_{\left(\alpha_{1}, \alpha_{2}\right)}$ are scalar coefficients that satisfy the conditions in Table 2.

Example 4.1. As an example, the expressions of the scalar coefficients $a_{\left(\alpha_{1}, \alpha_{2}\right)}, b_{\left(\alpha_{1}, \alpha_{2}\right)}, c_{\left(\alpha_{1}, \alpha_{2}\right)}$ and $d_{\left(\alpha_{1}, \alpha_{2}\right)}$ used in this paper are given as follows

$$
\begin{aligned}
& a_{\left(\alpha_{1}, \alpha_{2}\right)}=\alpha_{1}\left(1-\alpha_{2}\right), \\
& b_{\left(\alpha_{1}, \alpha_{2}\right)}=\alpha_{1} \alpha_{2}\left(\frac{\alpha_{1}+\alpha_{2}}{2}\right), \\
& c_{\left(\alpha_{1}, \alpha_{2}\right)}=\alpha_{2}\left(1-\alpha_{1}\right), \\
& d_{\left(\alpha_{1}, \alpha_{2}\right)}=\left(1-\alpha_{1}\right)\left(1-\alpha_{2}\right) .
\end{aligned}
$$

Their graphical representations are given in Figs. 5-8, respectively.

\section{Numerical Illustrative Examples}

To illustrate the interpolated characteristic of a memfractor between a memcapacitor, a memristor, a meminductor and second-order memristor we consider three numerical examples $\left(0 \leq \alpha_{1} \leq 1\right.$, $\left.\alpha_{2}=1\right),\left(\alpha_{1}=1,0 \leq \alpha_{2} \leq 1\right)$, and $\left(0 \leq \alpha_{1} \leq 1\right.$, $\left.0 \leq \alpha_{2} \leq 1\right)$.

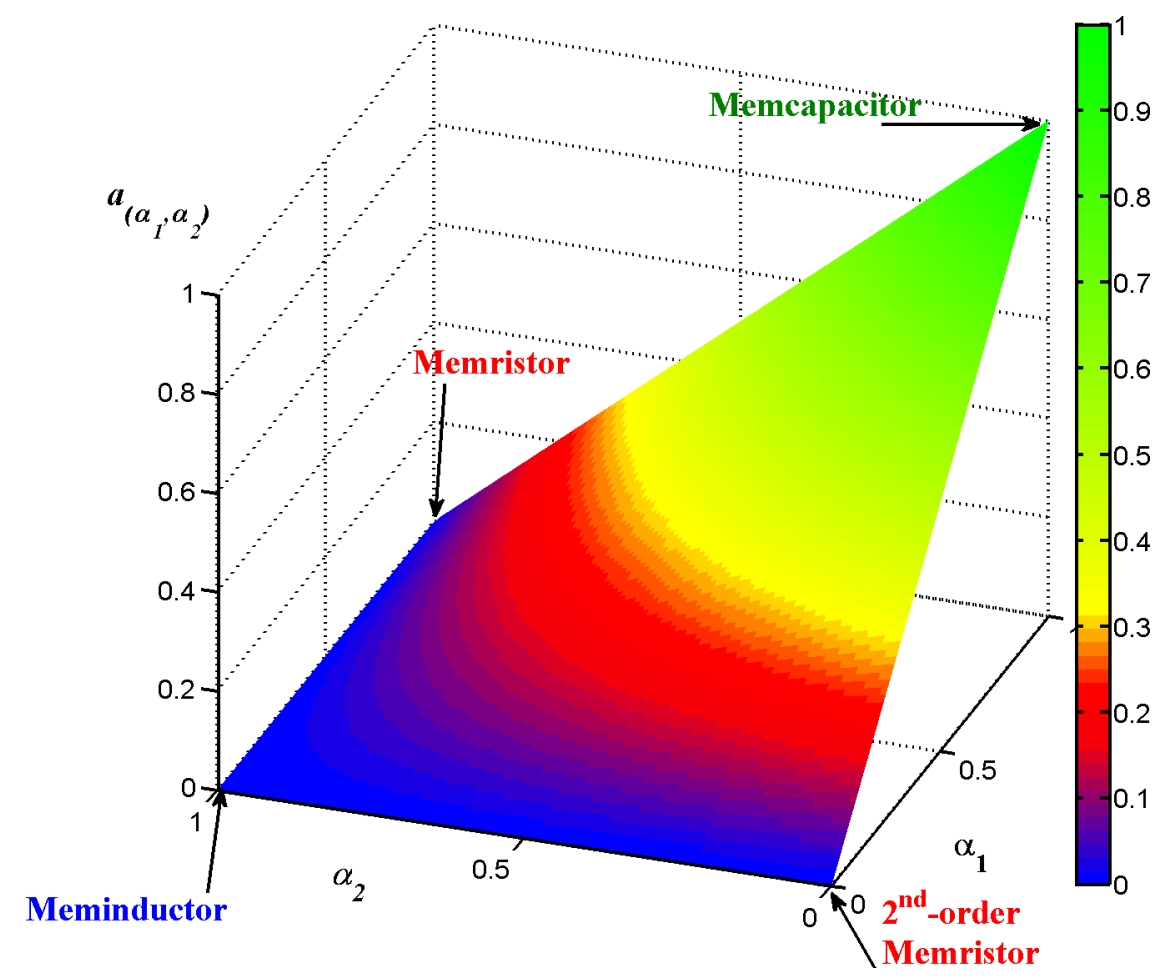

Fig. 5. Graphical representation of the coefficient $a_{\left(\alpha_{1}, \alpha_{2}\right)}=\alpha_{1}\left(1-\alpha_{2}\right)$. 


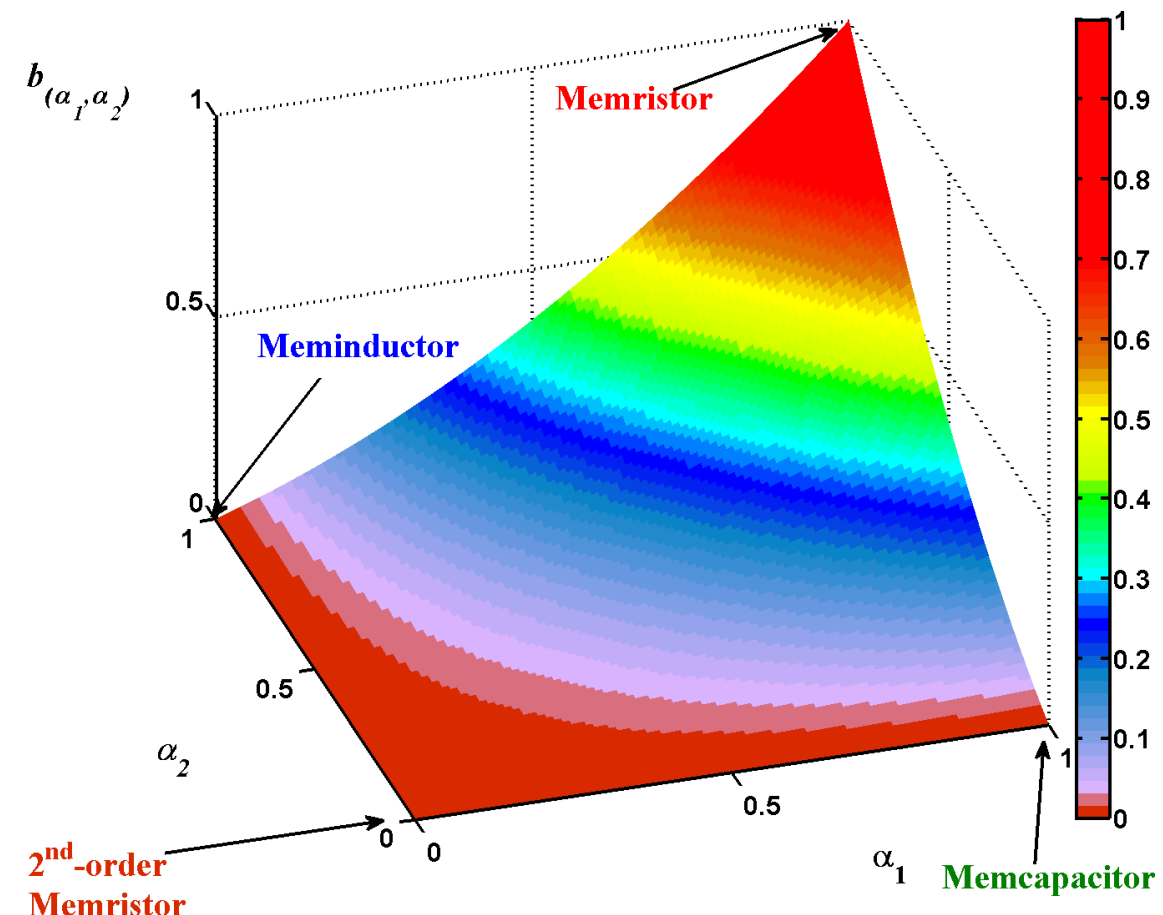

Fig. 6. Graphical representation of the coefficient $b_{\left(\alpha_{1}, \alpha_{2}\right)}=\alpha_{1} \alpha_{2} \frac{\left(\alpha_{1}+\alpha_{2}\right)}{2}$.

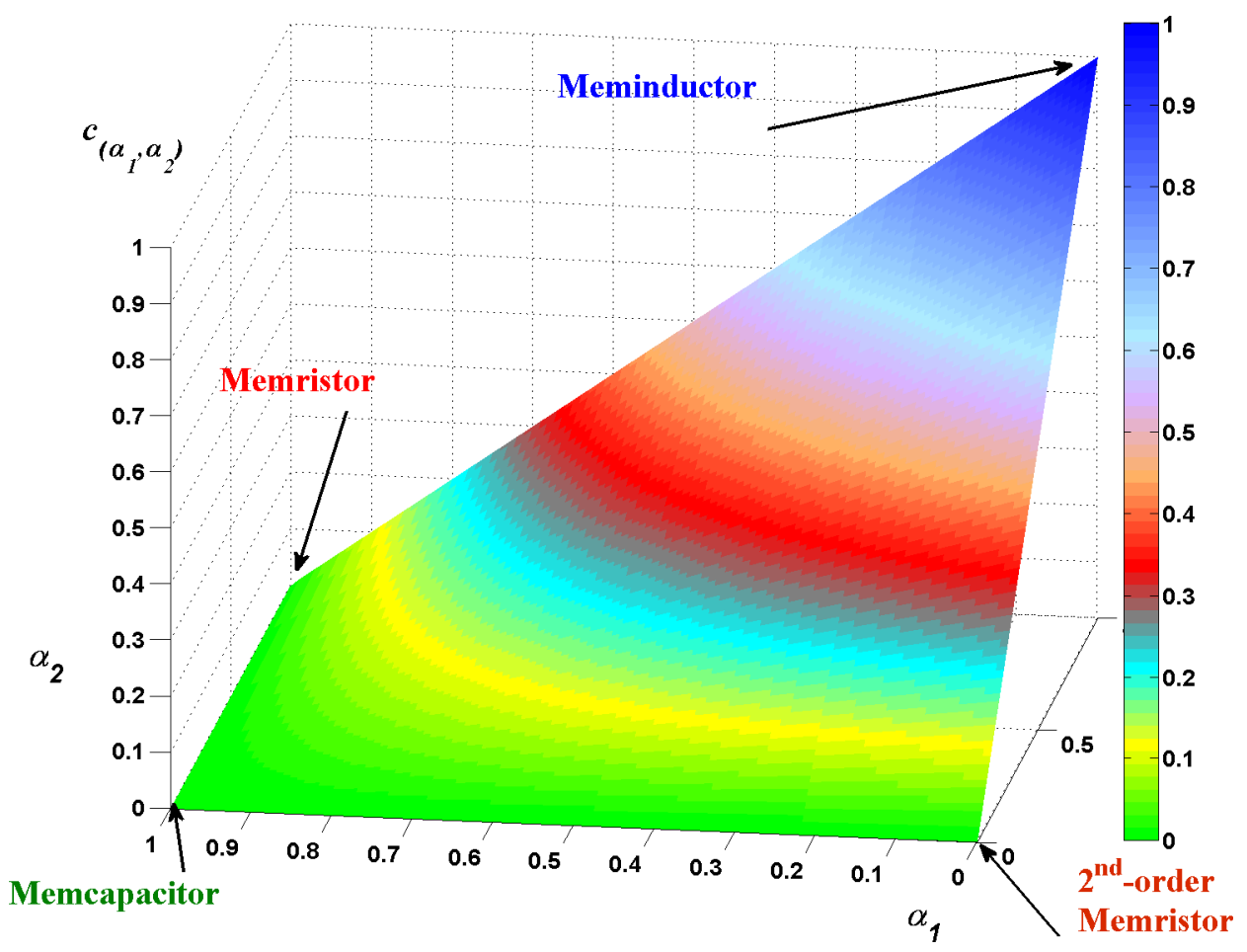

Fig. 7. Graphical representation of the coefficient $c_{\left(\alpha_{1}, \alpha_{2}\right)}=\alpha_{2}\left(1-\alpha_{1}\right)$. 


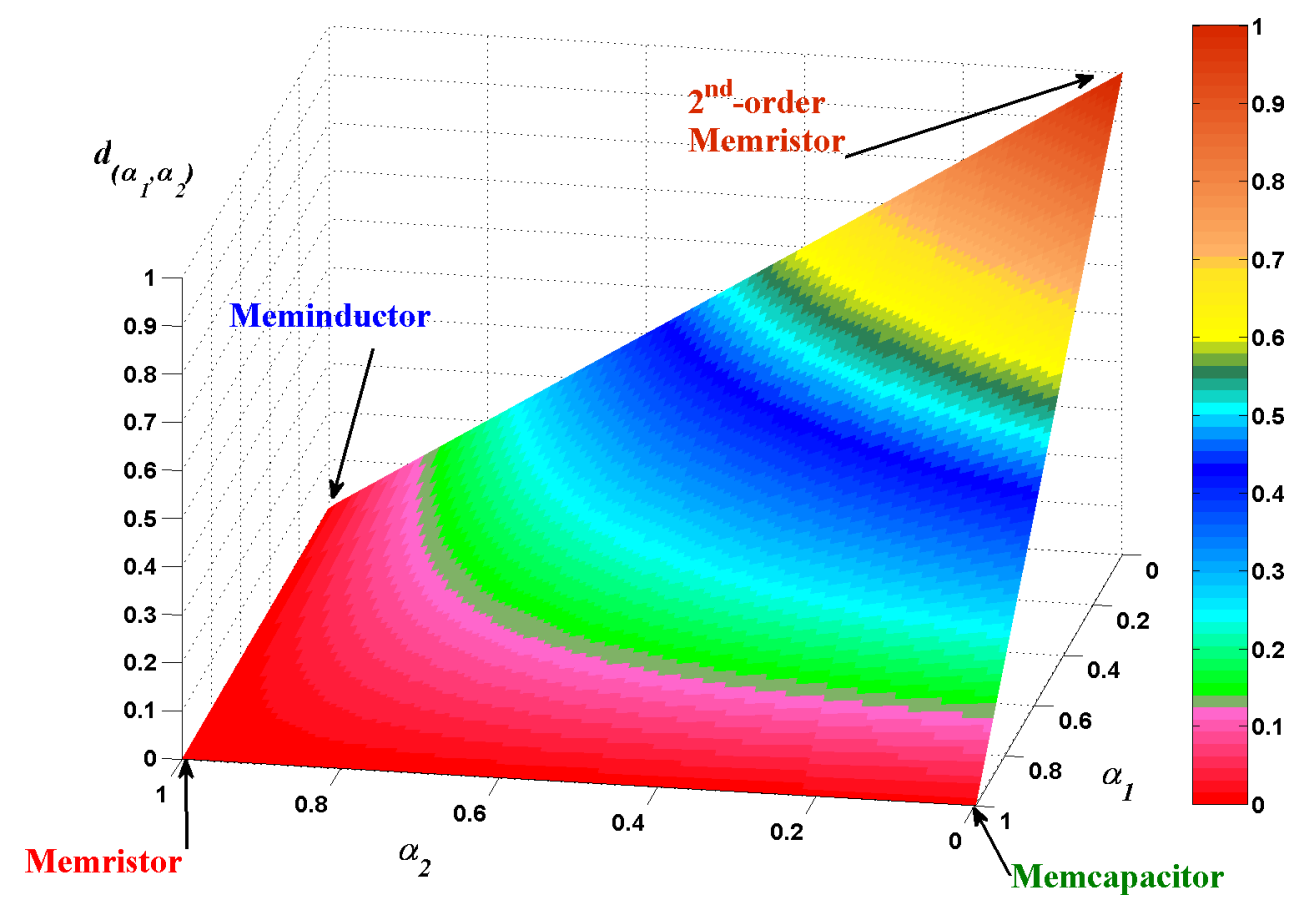

Fig. 8. Graphical representation of the coefficient $d_{\left(\alpha_{1}, \alpha_{2}\right)}=\left(1-\alpha_{1}\right)\left(1-\alpha_{2}\right)$.

Assuming that we have a memcapacitor with memcapacitance $^{1}$

$$
C_{M}(\sigma)=\frac{1}{1+(2+\sigma)^{2}},
$$

where $\sigma$ is the integral of charge $q$, namely $(\sigma(t)=$ $\left.\int_{-\infty}^{t} q(\tau) d \tau\right)$, a memristor with memresistance

$$
R_{M}(q)=1+q+q^{2}
$$

a meminductor with memductance

$$
L_{M}(q)=1+e^{q}
$$

and a second-order memristor with second-order memresistance

$$
R_{2 M}(\sigma)=1+\sigma+\sigma^{2} .
$$

We suppose that the current source $i(t)$ applied across the memfractor is given by

$$
\begin{cases}i(t)=\cos (t), & \text { if } t \geq 0 \\ 0, & \text { if } t<0\end{cases}
$$

and assuming the initial charge $q_{0}=q(0)=0$, then we obtain upon integrating (43) the corresponding charge

$$
q(t)=\int_{0}^{t} i(\tau) d \tau=\sin (t)
$$

and

$$
\sigma(t)=\int_{0}^{t} q(\tau) d \tau=1-\cos (t)
$$

substituting (44) into (33) we obtain the voltage across the memfractor, namely

$$
v(t)=D_{t}^{1-\alpha_{1}}\left(F_{M}^{\alpha_{1}, \alpha_{2}}(t) D_{t}^{\alpha_{2}}(\sin (t))\right) .
$$

For the calculation of fractional derivative, we use a numerical method based on GrünwaldLetnikov definition [see (7) and (8)], with a constant integration step size $h=0.002$.

\subsection{Intermediate behavior of memfractance between memristor and meminductor}

In this subsection, we illustrate the interpolated characteristic of memfractance between a memristor and a meminductor, therefore we consider the case $0 \leq \alpha_{1} \leq 1, \alpha_{2}=1$ (see Table 1 ).

\footnotetext{
${ }^{1}$ We have chosen the formula (39) for $C_{M}(\sigma(t))$ because it gives the $v_{C}-q$ curve as a simple loop passing through the origin similar to the one given in [Di Ventra et al., 2009].
} 


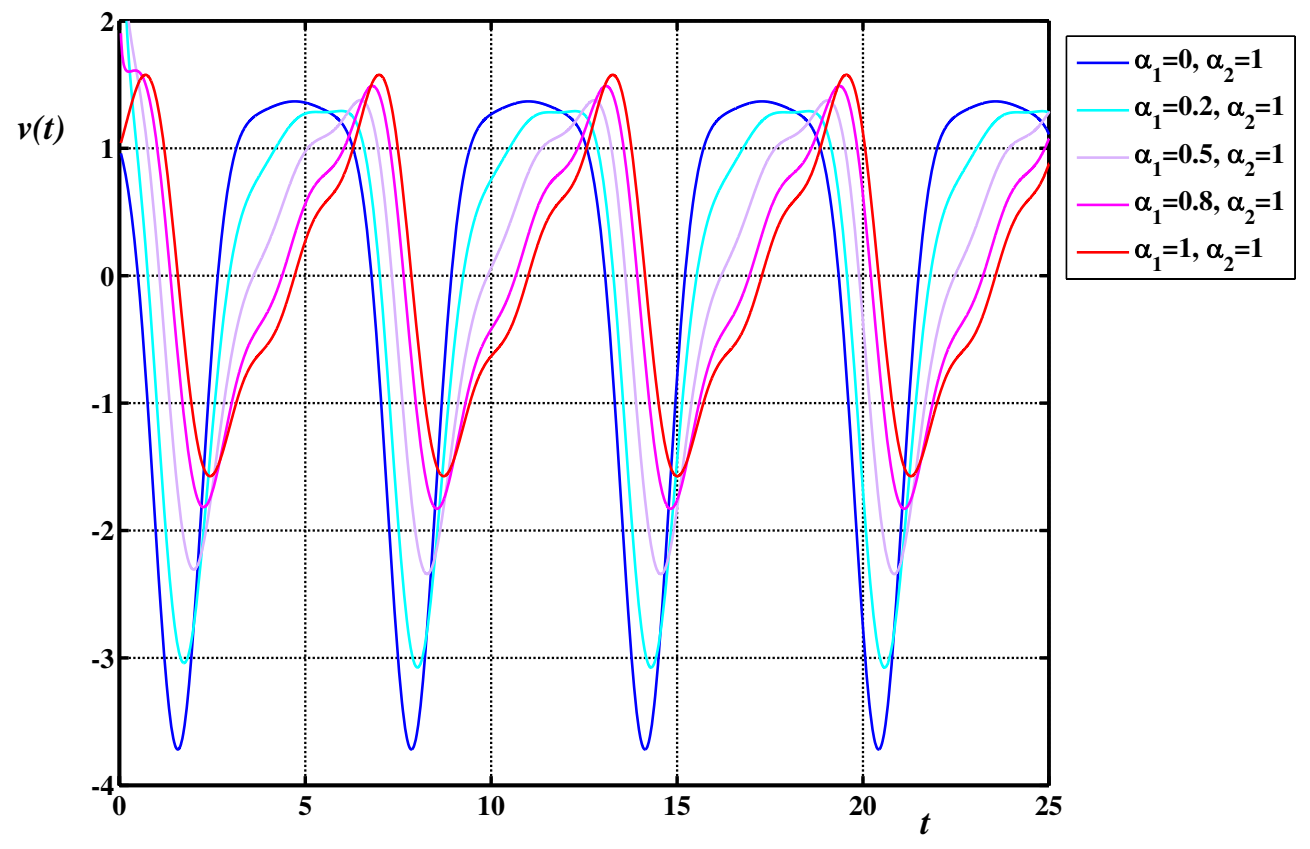

Fig. 9. Periodic waveform of $(v-t$ curve $)$ for $\left(0 \leq \alpha_{1} \leq 1, \alpha_{2}=1\right)$ obtained by solving $(33)$ with $F_{M}^{\alpha_{1}, \alpha_{2}}(t)=F_{M}^{\alpha_{1}, 1}(t)=$ $\alpha_{1}\left(\frac{1+\alpha_{1}}{2}\right)\left(1+\sin (t)+\sin ^{2}(t)\right)+\left(1-\alpha_{1}\right)\left(1+e^{\sin (t)}\right)$.

Figure 9 displays the time variation of voltage ( $v-t$ curve) which always has a periodic waveform.

Figure 10 shows the hysteresis loop (Lissajous curve) in the $v-i$ plane. For $\alpha_{1}=1, \alpha_{2}=1$ (memristor case) there is a pinched hysteresis loop: doublevalued Lissajous curve for all time $t$ except when it passes through the pinched point $(0,0)$. Upon decreasing $\alpha_{1}$ the pinched point is moved from $(0,0)$ and disappears when $\alpha_{1}$ passes the critical value $\alpha_{1} \approx 0.33$, then the hysteresis loop becomes clearly a double-valued Lissajous curve which changes its shape continuously and takes its final shape when $\left(\alpha_{1}=0, \alpha_{2}=1\right)$ corresponding to the hysteresis loop of a meminductor.

Figure 11 shows the hysteresis loop in the $v-q$ plane. When $\alpha_{1}=1$ and $\alpha_{2}=1$ (memristor case) the relation (46) becomes

$$
v(t)=R_{M}(q(t)) \cos (t),
$$

substituting (40) and (44) into (47) and taking into account that $\cos (t)=\mp \sqrt{1-\sin ^{2}(t)}$ yields

$$
\begin{aligned}
v(t) & =\mp\left(1+\sin (t)+\sin ^{2}(t)\right) \sqrt{1-\sin ^{2}(t)} \\
& =\mp\left(1+q(t)+q^{2}(t)\right) \sqrt{1-q^{2}(t)},
\end{aligned}
$$

which is a double-valued function of $q$, in this case, the hysteresis loop is a double-valued Lissajous curve symmetric with respect to $q$-axis. Upon decreasing the derivative order $\alpha_{1}$ from 1 to 0 , the loop changes continuously its shape and takes its final form when $\left(\alpha_{1}=0, \alpha_{2}=1\right)$ where it collapses into a single-valued function corresponding to the hysteresis loop of a meminductor, in this case the relation (46) becomes

$$
v(t)=\frac{d}{d t}\left(L_{M}(q(t)) \cos (t)\right)
$$

substituting (41) and (44) into (50) yields

$$
\begin{aligned}
v(t) & =\left(1-\sin (t)-\sin ^{2}(t)\right) e^{\sin (t)}-\sin (t) \\
& =\left(1-q(t)-q^{2}(t)\right) e^{q(t)}-q(t),
\end{aligned}
$$

which is a single-valued function of $q$.

Figure 12 shows the hysteresis loop in the $\varphi-i$ plane. For $\alpha_{1}=0, \alpha_{2}=1$ (meminductor case) we have

$$
\begin{aligned}
\varphi(t) & =\left(1+e^{\sin (t)}\right) \cos (t) \\
& =\left(1+e^{\mp \sqrt{1-\cos ^{2}(t)}}\right) \cos (t) \\
& =\left(1+e^{\mp \sqrt{1-i^{2}(t)}}\right) i(t),
\end{aligned}
$$

which is a double-valued function of $i(t)$ for all time $t$ except when $i(t)=0$, so the hysteresis loop is a double-valued Lissajous curve for all time $t$ except when it passes through the pinched point $(0,0)$. 


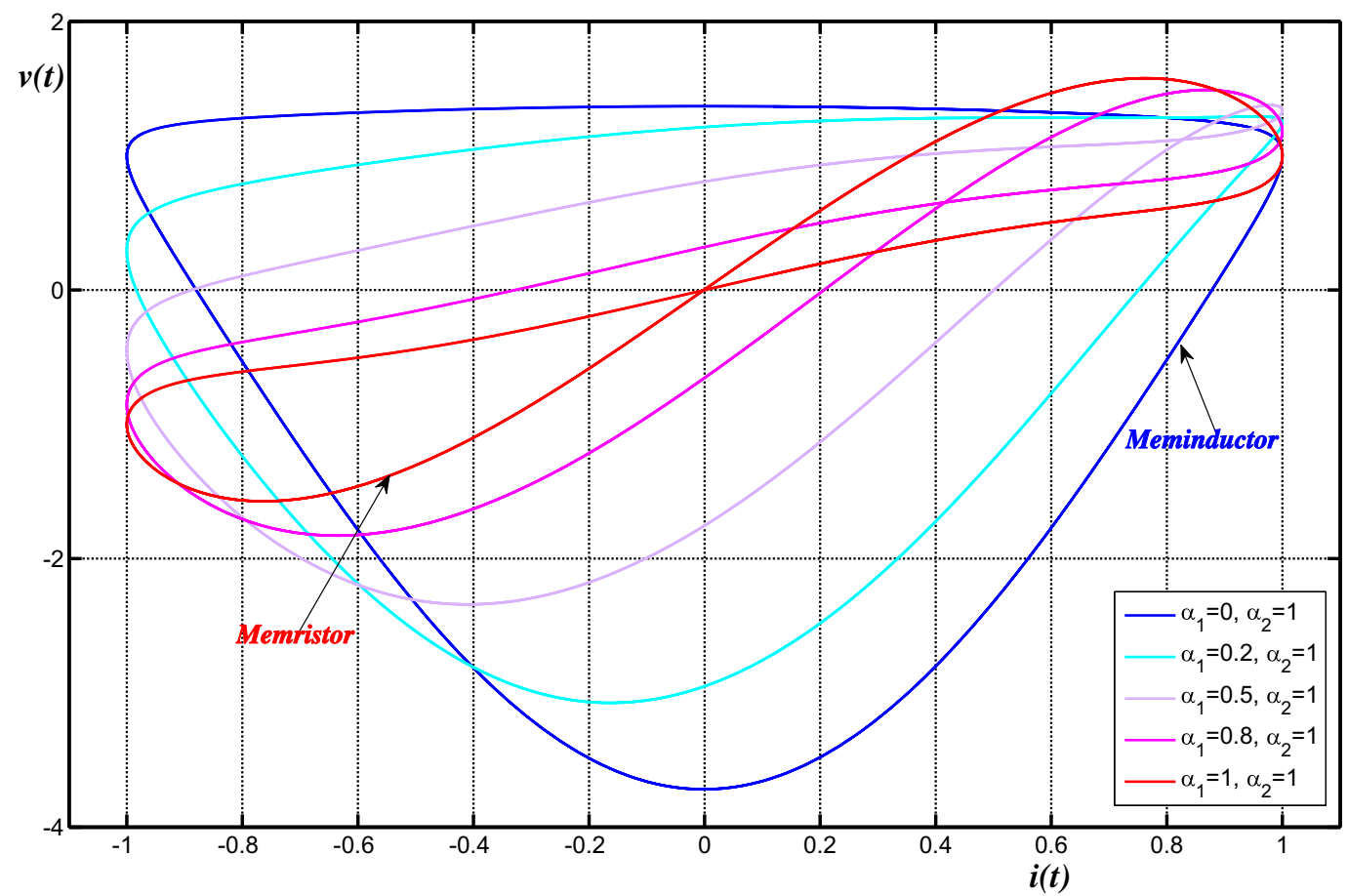

Fig. 10. Hysteresis loop (Lissajous curve) associated with Fig. 9 (Example 5.1) in the $v-i$ plane for $\left(0 \leq \alpha_{1} \leq 1, \alpha_{2}=1\right)$.

Upon increasing the derivative order $\alpha_{1}$ from 0 to 1 , the loop changes continuously its shape by moving its pinched point and when passing the critical value $\alpha_{1} \approx 0.32$, the pinched point disappears and the loop becomes a double-valued Lissajous curve of $\varphi(t)-i(t)$ for all time $t$ and takes its final form when $\left(\alpha_{1}=1, \alpha_{2}=1\right)$ corresponding to the hysteresis loop of a memristor.

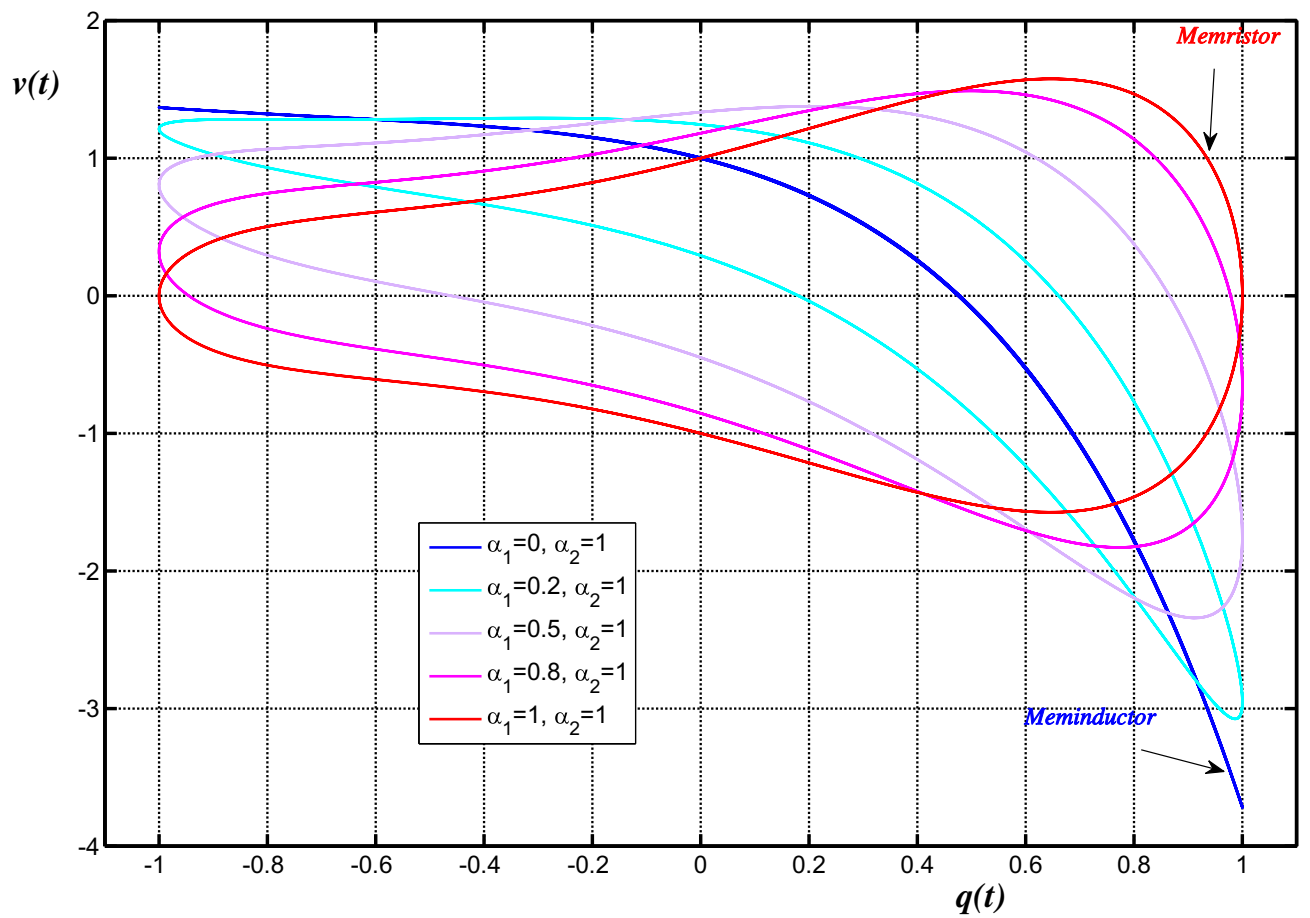

Fig. 11. Hysteresis loop (Lissajous curve) associated with Fig. 9 (Example 5.1) in the $v-q$ plane for $0 \leq \alpha_{1} \leq 1, \alpha_{2}=1$. 


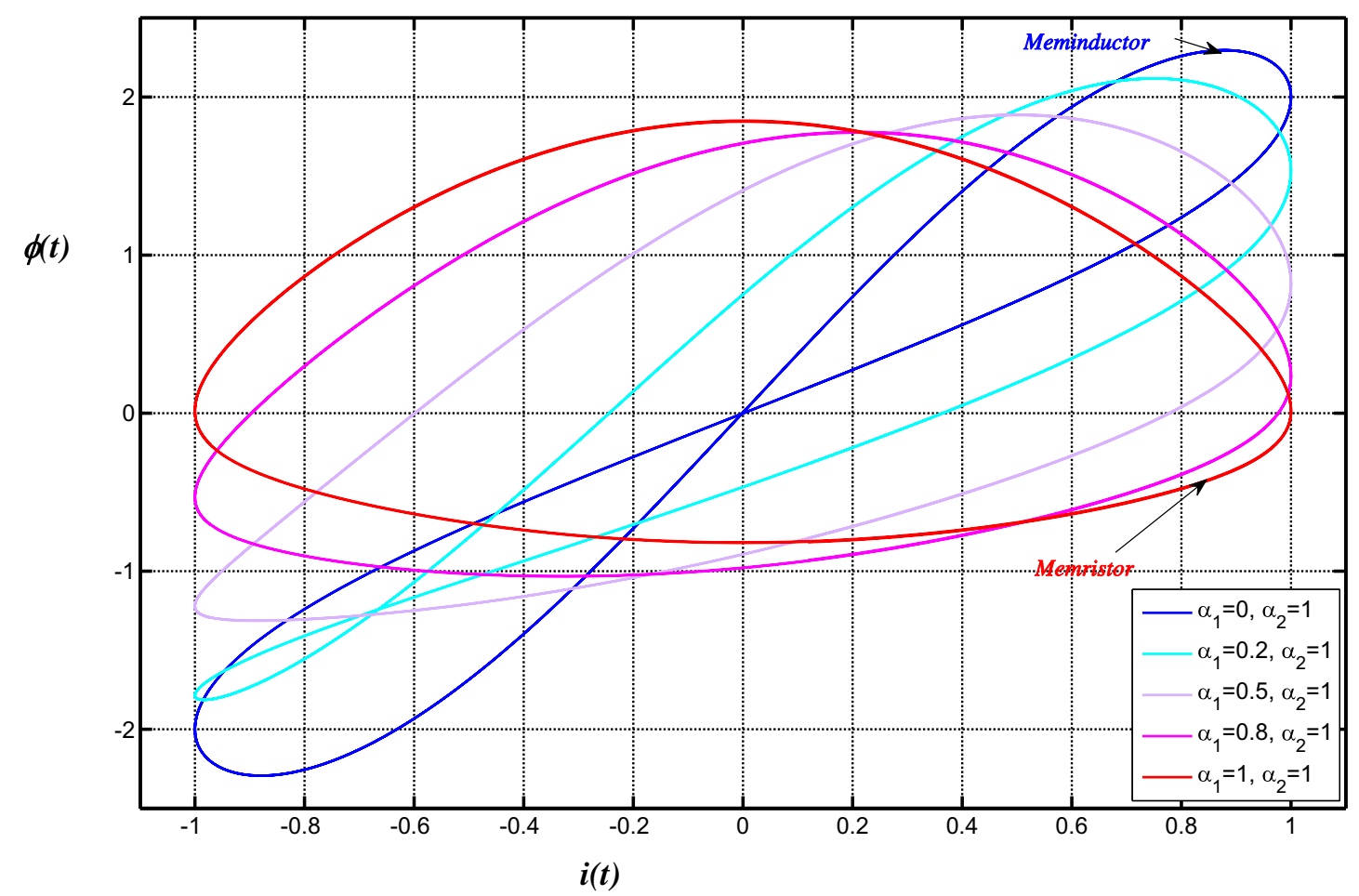

Fig. 12. Hysteresis loop (Lissajous curve) (Example 5.1) in the $\varphi-i$ plane for $0 \leq \alpha_{1} \leq 1, \alpha_{2}=1$.

Figure 13 shows the hysteresis loop (Lissajous curve) in the $\varphi-q$ plane. For $\alpha_{1}=1, \alpha_{2}=1$ (memristor case) we have

$$
\frac{d \varphi(t)}{d t}=\left(1+q(t)+q^{2}(t)\right) \frac{d q(t)}{d t}
$$

integrating with respect to $t$ gives

$$
\varphi(t)=q(t)+\frac{q^{2}(t)}{2}+\frac{q^{3}(t)}{3},
$$

which is a single-valued function of $q$. Upon decreasing $\alpha_{1}$, this hysteresis loop becomes a double-valued Lissajous curve which changes its shape continuously and takes its final shape when $\left(\alpha_{1}=0\right.$, $\alpha_{2}=1$ ) corresponding to the hysteresis loop of a meminductor, in this case, we have

$$
\varphi(t)=\mp\left(1+e^{q(t)}\right) \sqrt{1-q^{2}(t)},
$$

which is a double-valued function of $q$.

From this subsection, we postulate that any intermediate state between meminductor and memristor can be modeled by a memfractor element, choosing a convenient fractional derivative order $\alpha_{1}$ between 0 and 1 .

\subsection{Continuous behavior of memfractance between memristor and memcapacitor}

In this subsection, we illustrate the interpolated characteristic of a memfractor between a memristor and a memcapacitor. In particular, let us consider the case $\left(\alpha_{1}=1,0 \leq \alpha_{2} \leq 1\right)$.

Figure 14 displays the time variation of voltage ( $v$ - $t$ curve) which always has a periodic waveform. Figure 15 shows the hysteresis loop (Lissajous curve) in the $v-i$ plane. For $\alpha_{1}=1, \alpha_{2}=1$ (memristor case) the hysteresis loop is pinched at the origin point $(0,0)$ as mentioned in Sec. 5.1. Upon decreasing $\alpha_{2}$, this hysteresis loop becomes a double-valued Lissajous curve which changes its shape continuously and takes its final shape when $\left(\alpha_{1}=1\right.$, $\alpha_{2}=0$ ) corresponding to the hysteresis loop of a memcapacitor, in this case we have

$$
\begin{aligned}
v(t) & =\left(1+(3-\cos (t))^{2}\right) \sin (t) \\
& = \pm\left(1+(3-\cos (t))^{2}\right) \sqrt{1-\cos ^{2}(t)} \\
& = \pm\left(1+(3-i(t))^{2}\right) \sqrt{1-i^{2}(t)},
\end{aligned}
$$

which is a double-valued function of $i$.

Figure 16 shows the hysteresis loop in the $v-q$ plane. For $\alpha_{1}=1, \alpha_{2}=0$ we have a pinched 


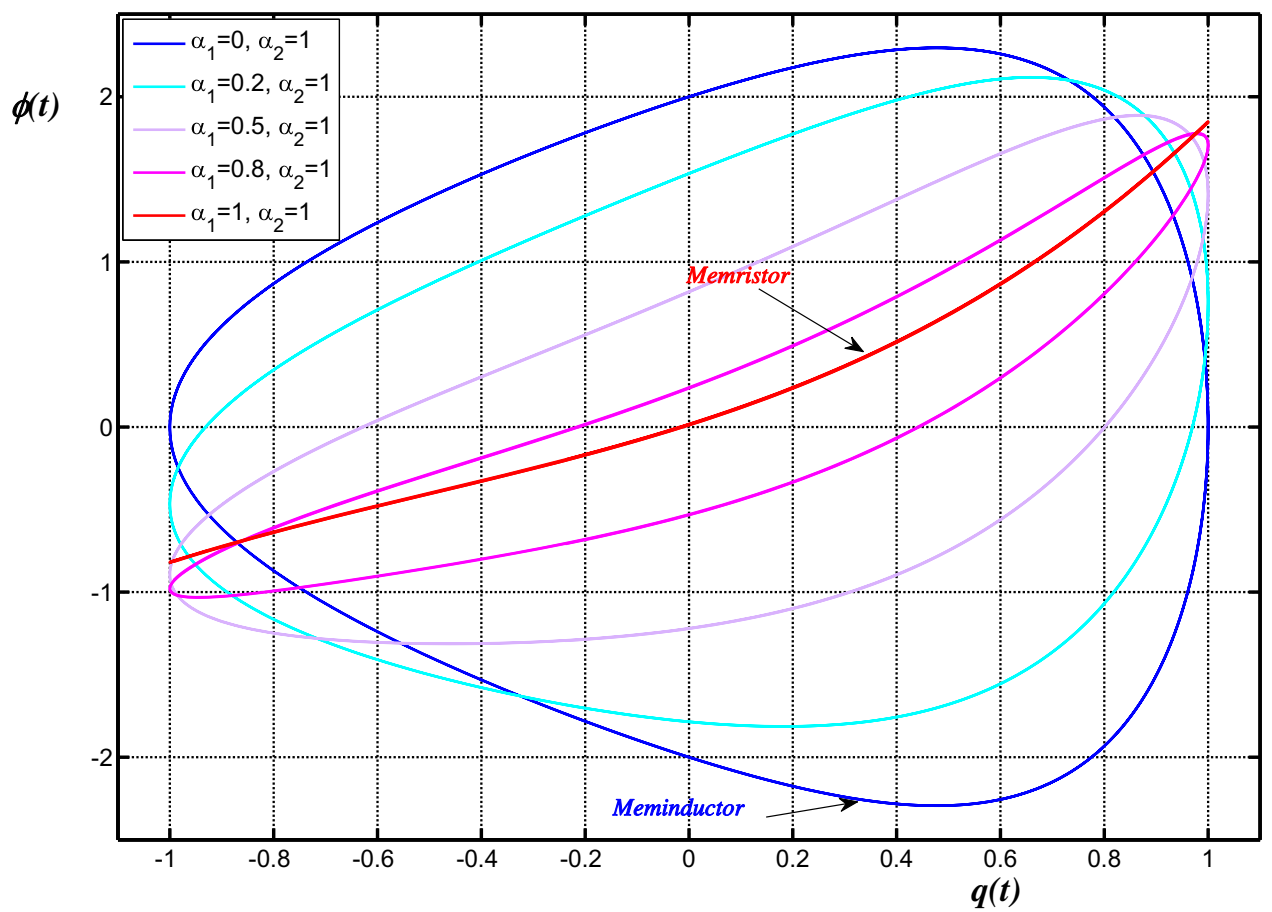

Fig. 13. Hysteresis loop (Lissajous curve) (Example 5.1) in the $\varphi-q$ plane for $\left(0 \leq \alpha_{1} \leq 1, \alpha_{2}=1\right)$.

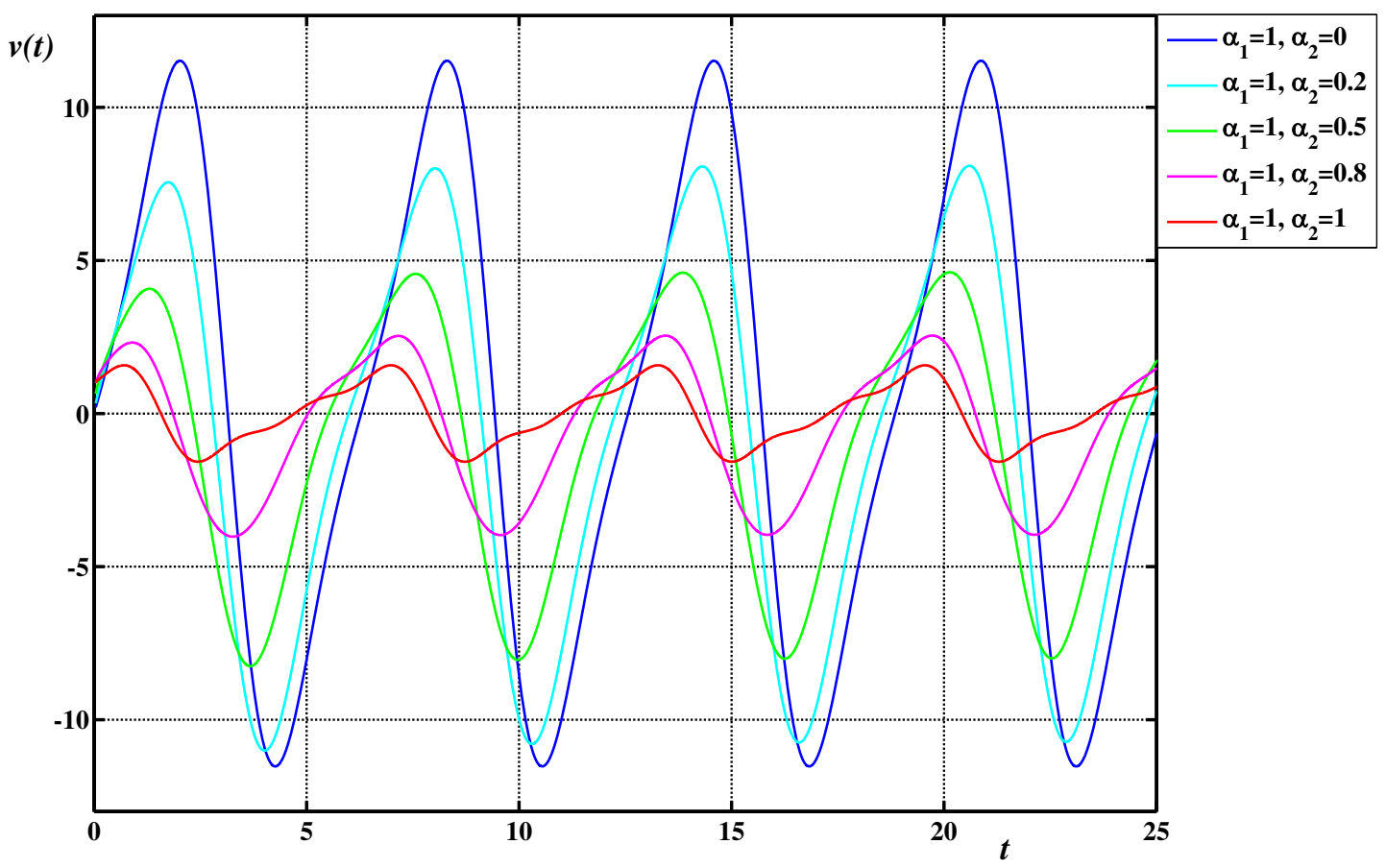

Fig. 14. Periodic waveform of ( $v-t$ curve) for $\left(\alpha_{1}=1,0 \leq \alpha_{2} \leq 1\right)$ obtained by solving (33) with $F_{M}^{\alpha_{1}, \alpha_{2}}(t)=F_{M}^{1, \alpha_{2}}(t)=$ $\left(1-\alpha_{2}\right)\left(1+\left(2+\int_{0}^{t} \sin (\tau) d \tau\right)^{2}\right)+\alpha_{2}\left(\frac{1+\alpha_{2}}{2}\right)\left(1+\sin (t)+\sin ^{2}(t)\right)$. 


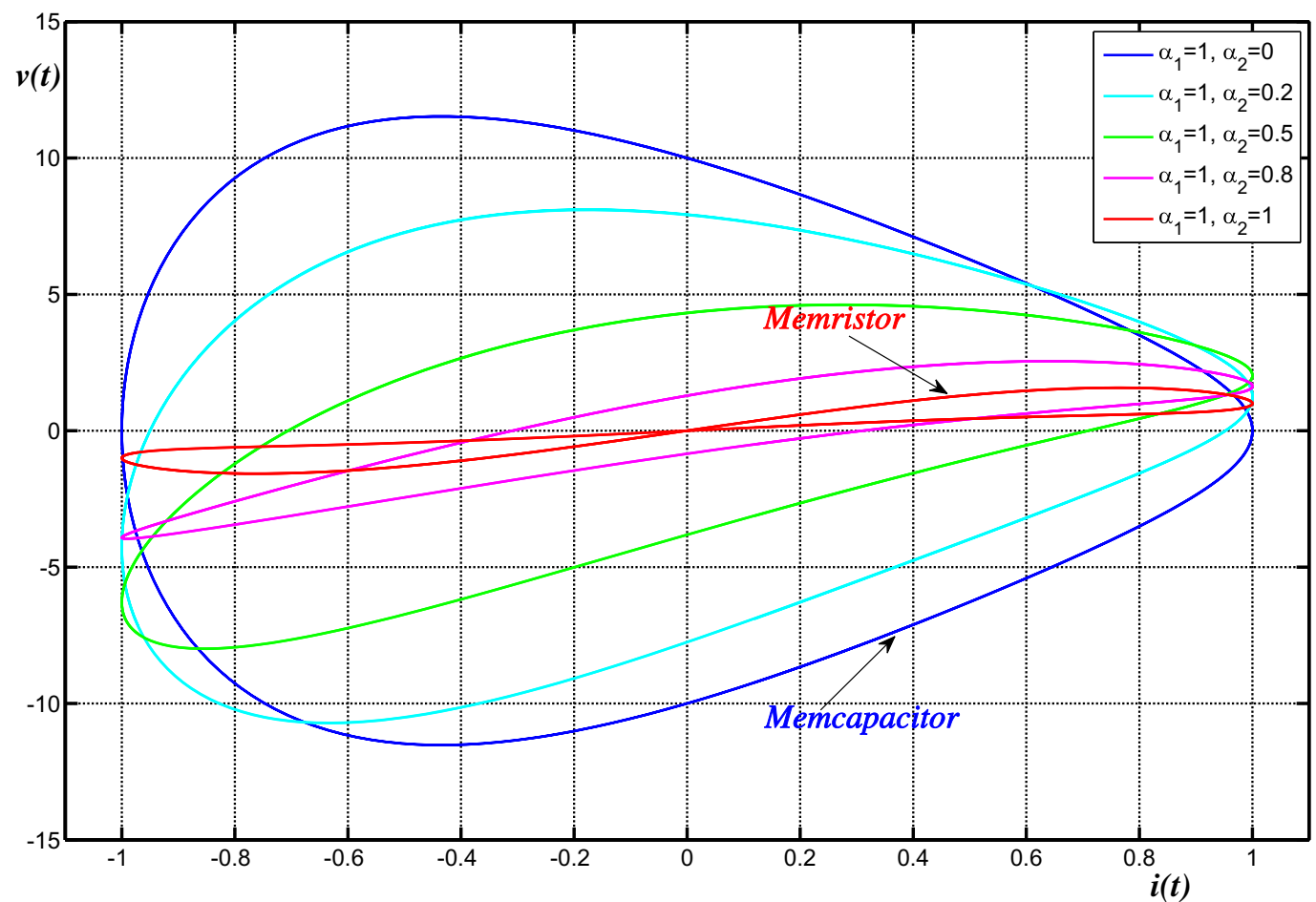

Fig. 15. Hysteresis loop (Lissajous curve) associated with Fig. 14 (Example 5.2) in the $v-i$ plane for $\left(\alpha_{1}=1,0 \leq \alpha_{2} \leq 1\right)$.

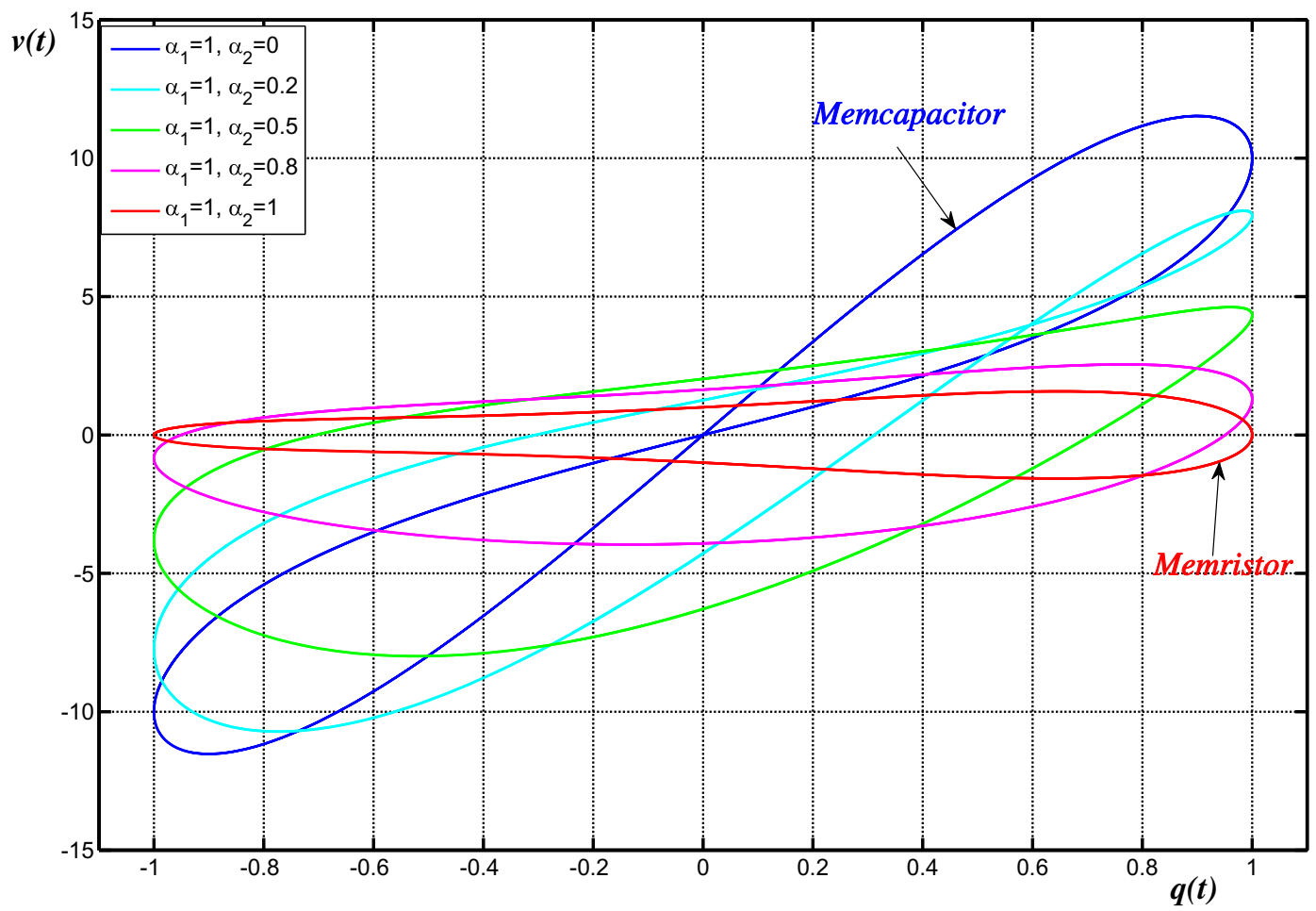

Fig. 16. Hysteresis loop (Lissajous curve) associated with Fig. 14 (Example 5.2) in the $v-q$ plane for $\left(\alpha_{1}=1,0 \leq \alpha_{2} \leq 1\right)$. 
hysteresis loop: double-valued Lissajous curve of $v(t)-q(t)$ for all time $t$ except when it passes through the origin $(0,0)$, where the loop is pinched (memcapacitor case) which agrees with the result obtained in [Di Ventra et al., 2009], in this case we have

$$
\begin{aligned}
v(t) & =\left(1+(3-\cos (t))^{2}\right) \sin (t) \\
& =\left(1+\left(3 \mp \sqrt{1-\sin ^{2}(t)}\right)^{2}\right) \sin (t) \\
& =\left(1+\left(3 \mp \sqrt{1-q^{2}(t)}\right)^{2}\right) q(t),
\end{aligned}
$$

which is a double-valued function of $q(t)$ for all time $t$ except when $q(t)=0$. Upon increasing the derivative order $\alpha_{2}$, the loop changes its shape by moving its pinched point and when passing the critical value $\alpha_{2} \approx 0.35$, the pinched point disappears and the loop becomes a double-valued Lissajous curve of $v(t)-q(t)$ for all time $t$.

Figure 17 displays the time variation of flux $(\varphi-$ $t$ curve) which has an oscillation form. For $\alpha_{1}=1$, $\alpha_{2}=0$ (memcapacitor case) the curve has a periodic waveform in a horizontal band but when $\alpha_{2}$ increases the band of oscillations inclines (Fig. 20 shows the variation of the slope of this band when varying $\alpha_{2}$ ) and for $\alpha_{2}=1$ the band of oscillation becomes horizontal. In order to investigate this phenomenon, we calculate $\varphi(t)$ by integrating the relation (33) and taking into account that the fractional derivative of the sine function ${ }_{0} D_{t}^{\alpha_{2}} \sin (t)$ is not an exact periodic function [Tavazoei, 2010] but it converges rapidly to the periodic function $\sin \left(t+\alpha_{2} \frac{\pi}{2}\right)$ as illustrated in Fig. 18. Considering that

$$
{ }_{0} D_{t}^{\alpha_{2}} \sin (t) \approx \sin \left(t+\alpha_{2} \frac{\pi}{2}\right), \text { for } t \gg 0
$$

we can write

$$
{ }_{0} D_{t}^{\alpha_{2}} \sin (t)=\sin \left(t+\alpha_{2} \frac{\pi}{2}\right)+\varepsilon(t),
$$

where $\lim _{t \rightarrow+\infty} \varepsilon(t)=0$, and we have

$$
\begin{aligned}
v(t)= & F_{M}^{1, \alpha_{2}}(t) D_{t}^{\alpha_{2}} \sin (t) \\
= & \left(\left(1-\alpha_{2}\right)\left(1+(3-\cos (t))^{2}\right)+\alpha_{2}\left(\frac{1+\alpha_{2}}{2}\right)\right. \\
& \left.\times\left(1+\sin (t)+\sin ^{2}(t)\right)\right) D_{t}^{\alpha_{2}} \sin (t) \\
= & \tilde{v}(t)+\xi(t),
\end{aligned}
$$

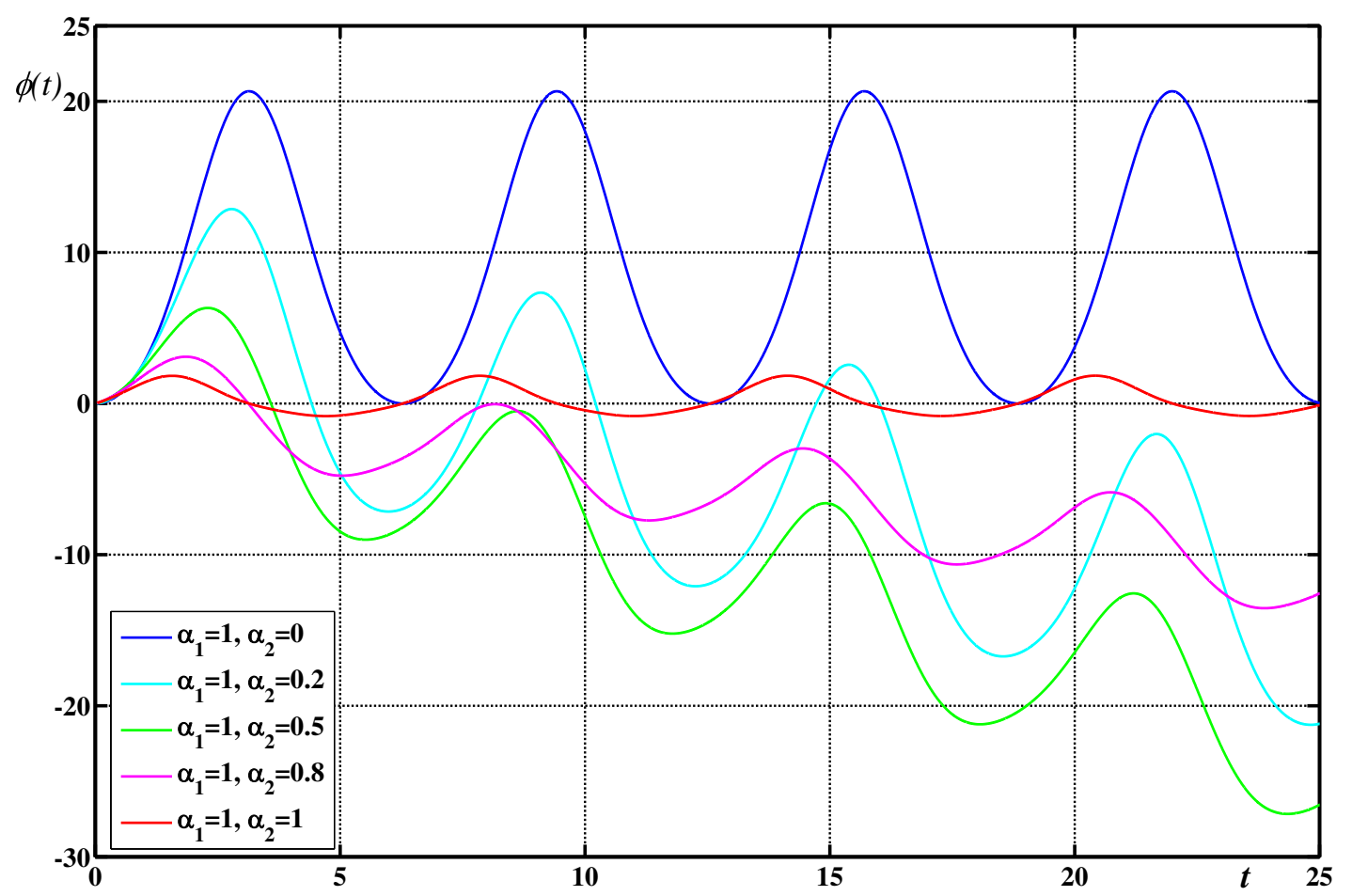

Fig. 17. Oscillation-form of ( $\varphi-t$ curve) associated with Fig. 14 (Example 5.2) for $\left(\alpha_{1}=1,0 \leq \alpha_{2} \leq 1\right)$. 


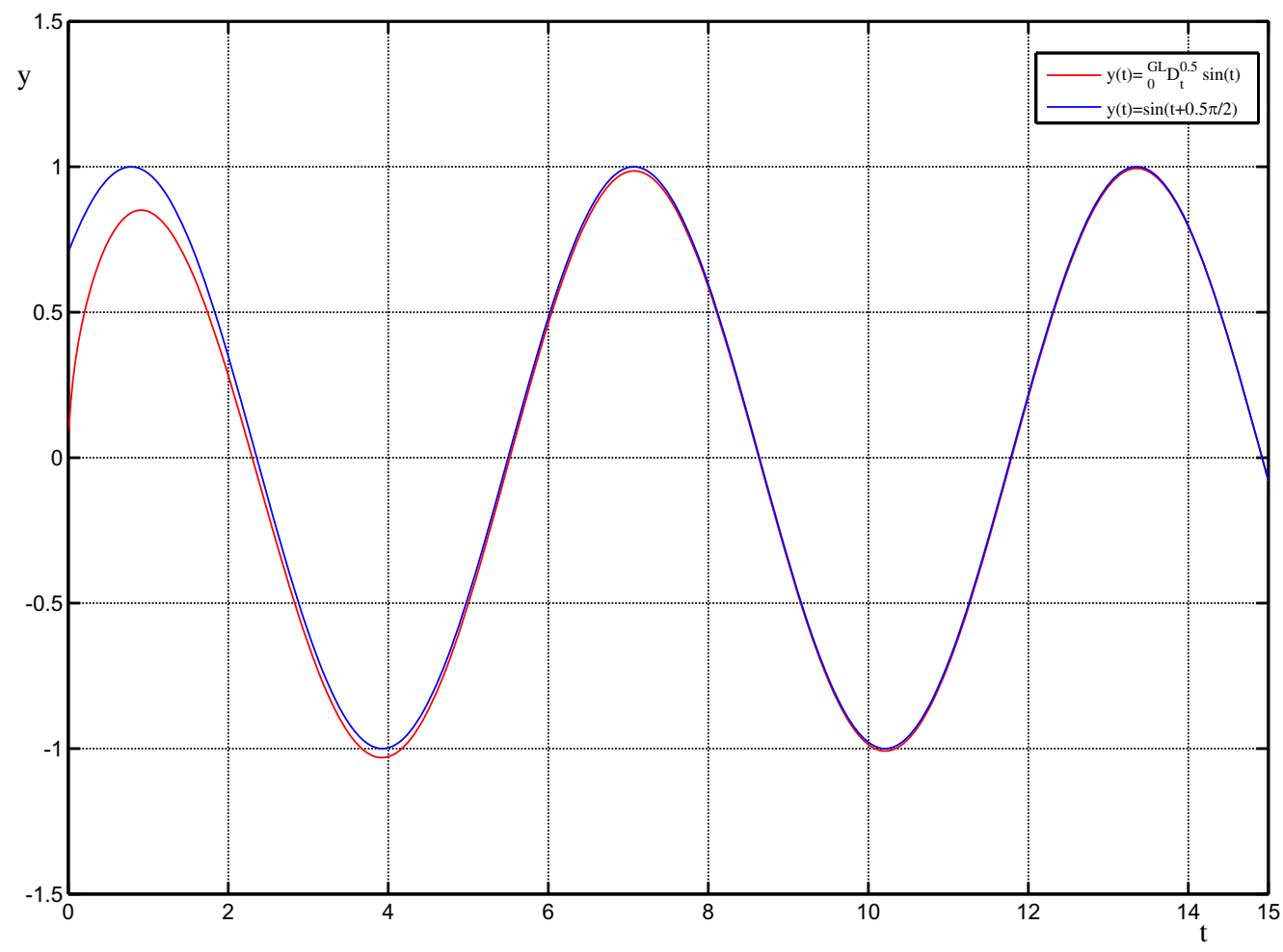

Fig. 18. The half derivative of sine function compared with the function $\sin \left(t+0.5 \frac{\pi}{2}\right)$.

where

$$
\begin{aligned}
\tilde{v}(t)= & \left(\left(1-\alpha_{2}\right)\left(1+(3-\cos (t))^{2}\right)\right. \\
& \left.+\alpha_{2}\left(\frac{1+\alpha_{2}}{2}\right)\left(1+\sin (t)+\sin ^{2}(t)\right)\right) \\
& \times \sin \left(t+\alpha_{2} \frac{\pi}{2}\right)
\end{aligned}
$$

and

$$
\begin{aligned}
\xi(t)= & F_{M}^{1, \alpha_{2}}(t) \varepsilon(t)=\left(\left(1-\alpha_{2}\right)\left(1+(3-\cos (t))^{2}\right)\right. \\
& \left.+\alpha_{2}\left(\frac{1+\alpha_{2}}{2}\right)\left(1+\sin (t)+\sin ^{2}(t)\right)\right) \varepsilon(t) .
\end{aligned}
$$

Since

$$
\begin{aligned}
F_{M}^{1, \alpha_{2}}(t)= & \left(\left(1-\alpha_{2}\right)\left(1+(3-\cos (t))^{2}\right)\right. \\
& \left.+\alpha_{2}\left(\frac{1+\alpha_{2}}{2}\right)\left(1+\sin (t)+\sin ^{2}(t)\right)\right)
\end{aligned}
$$

is a periodic bounded function of period $2 \pi$ and $\varepsilon(t)$ is rapidly vanishing, we have

$$
\lim _{t \rightarrow+\infty} \xi(t)=0 .
$$

Taking into account the classical trigonometric relations we obtain

$$
\begin{aligned}
\tilde{v}(t)= & \left(\left(1-\alpha_{2}\right)\left(1+(3-\cos (t))^{2}\right)\right. \\
& \left.+\alpha_{2}\left(\frac{1+\alpha_{2}}{2}\right)\left(1+\sin (t)+\sin ^{2}(t)\right)\right) \\
& \times\left(\sin (t) \cos \left(\alpha_{2} \frac{\pi}{2}\right)+\sin \left(\alpha_{2} \frac{\pi}{2}\right) \cos (t)\right) \\
= & -\left(\frac{\alpha_{2}^{2}+3 \alpha_{2}-2}{8}\right) \sin \left(3 t+\frac{\alpha_{2} \pi}{2}\right) \\
& +3\left(\alpha_{2}-1\right) \sin \left(2 t+\frac{\alpha_{2} \pi}{2}\right)-\frac{\alpha_{2}\left(\alpha_{2}+1\right)}{4} \\
& \times \cos \left(2 t+\frac{\alpha_{2} \pi}{2}\right)+\frac{3 \alpha_{2}^{2}-39 \alpha_{2}+42}{4} \\
& \times \sin \left(t+\frac{\alpha_{2} \pi}{2}\right)+\frac{\alpha_{2}^{2}+3 \alpha_{2}-2}{8} \\
& \times \sin \left(t-\frac{\alpha_{2} \pi}{2}\right)+\left[\frac{\alpha_{2}\left(\alpha_{2}+1\right)}{4} \cos \left(\frac{\alpha_{2} \pi}{2}\right)\right. \\
& \left.+3\left(\alpha_{2}-1\right) \sin \left(\frac{\alpha_{2} \pi}{2}\right)\right] \\
= & P(t)+A,
\end{aligned}
$$


where

$$
\begin{aligned}
P(t)= & -\left(\frac{\alpha_{2}^{2}+3 \alpha_{2}-2}{8}\right) \sin \left(3 t+\frac{\alpha_{2} \pi}{2}\right) \\
& +3\left(\alpha_{2}-1\right) \sin \left(2 t+\frac{\alpha_{2} \pi}{2}\right)-\frac{\alpha_{2}\left(\alpha_{2}+1\right)}{4} \\
& \times \cos \left(2 t+\frac{\alpha_{2} \pi}{2}\right)+\frac{3 \alpha_{2}^{2}-39 \alpha_{2}+42}{4} \\
& \times \sin \left(t+\frac{\alpha_{2} \pi}{2}\right)+\frac{\alpha_{2}^{2}+3 \alpha_{2}-2}{8} \\
& \times \sin \left(t-\frac{\alpha_{2} \pi}{2}\right),
\end{aligned}
$$

which is a bounded periodic function in $t$ with period $2 \pi$ and

$A=\frac{\alpha_{2}\left(\alpha_{2}+1\right)}{4} \cos \left(\frac{\alpha_{2} \pi}{2}\right)+3\left(\alpha_{2}-1\right) \sin \left(\frac{\alpha_{2} \pi}{2}\right)$,

is a constant. Then

$$
v(t)=P(t)+A+\xi(t)
$$

this means that the voltage waveform converges to a periodic function plus a constant, which can be interpreted as connecting a battery of a constant voltage in series with the periodic component. Now setting

$$
\tilde{\varphi}(t)=\int_{0}^{t} \tilde{v}(\tau) d \tau
$$

and taking into account relation (53) we obtain

$$
\begin{aligned}
\tilde{\varphi}(t)= & A t+\int_{0}^{t} P(\tau) d \tau \\
= & \left(\frac{\alpha_{2}^{2}+3 \alpha_{2}-2}{24}\right) \cos \left(3 t+\frac{\alpha_{2} \pi}{2}\right) \\
& -\frac{3\left(\alpha_{2}-1\right)}{2} \cos \left(2 t+\frac{\alpha_{2} \pi}{2}\right)-\frac{\alpha_{2}\left(\alpha_{2}+1\right)}{8} \\
& \times \sin \left(2 t+\frac{\alpha_{2} \pi}{2}\right)+\frac{-3 \alpha_{2}^{2}+39 \alpha_{2}-42}{4} \\
& \times \cos \left(t+\frac{\alpha_{2} \pi}{2}\right)+\frac{-\alpha_{2}^{2}-3 \alpha_{2}+2}{8} \\
& \times \cos \left(t-\frac{\alpha_{2} \pi}{2}\right)+A t \\
& +\left[\frac{5 \alpha_{2}^{2}-48 \alpha_{2}+53}{6} \cos \left(\frac{\alpha_{2} \pi}{2}\right)\right. \\
& \left.+\frac{\alpha_{2}\left(\alpha_{2}+1\right)}{8} \sin \left(\frac{\alpha_{2} \pi}{2}\right)\right] \\
= & f(t)+A t+B
\end{aligned}
$$

where

$$
\begin{aligned}
f(t)= & \left(\frac{\alpha_{2}^{2}+3 \alpha_{2}-2}{24}\right) \cos \left(3 t+\frac{\alpha_{2} \pi}{2}\right) \\
& -\frac{3\left(\alpha_{2}-1\right)}{2} \cos \left(2 t+\frac{\alpha_{2} \pi}{2}\right)-\frac{\alpha_{2}\left(\alpha_{2}+1\right)}{8} \\
& \times \sin \left(2 t+\frac{\alpha_{2} \pi}{2}\right)+\frac{-3 \alpha_{2}^{2}+39 \alpha_{2}-42}{4} \\
& \times \cos \left(t+\frac{\alpha_{2} \pi}{2}\right)+\frac{-\alpha_{2}^{2}-3 \alpha_{2}+2}{8} \\
& \times \cos \left(t-\frac{\alpha_{2} \pi}{2}\right),
\end{aligned}
$$

which is a bounded periodic function in $t$ with period $2 \pi$, and

$$
\begin{aligned}
B= & \frac{5 \alpha_{2}^{2}-48 \alpha_{2}+53}{6} \cos \left(\frac{\alpha_{2} \pi}{2}\right) \\
& +\frac{\alpha_{2}\left(\alpha_{2}+1\right)}{8} \sin \left(\frac{\alpha_{2} \pi}{2}\right),
\end{aligned}
$$

is a constant. Since the graphs of sine and cosine functions are both symmetric with respect to the axe of abscissas then the graph of $f$ will be symmetric with respect to the axe of abscissas too, so we have

$$
\max _{t \geq 0}(f(t))=-\min _{t \geq 0}(f(t))
$$

then

$$
\begin{aligned}
A t+ & B-\max _{t \geq 0}(f(t)) \\
& \leq \tilde{\varphi}(t) \leq A t+B+\max _{t \geq 0}(f(t)),
\end{aligned}
$$

setting

$$
B_{\min }=B-\max _{t \geq 0}(f(t))
$$

and

$$
B_{\max }=B+\max _{t \geq 0}(f(t)),
$$

SO

$$
A t+B_{\min } \leq \tilde{\varphi}(t) \leq A t+B_{\max },
$$

then the graph $\gamma_{\tilde{\varphi}}$ of $\tilde{\varphi}$ oscillates around the straight line

$$
\Delta: y=A t+B
$$

as shown in Fig. 19, where its local minima lie in the straight line

$$
T_{\min }: y=A t+B_{\min },
$$




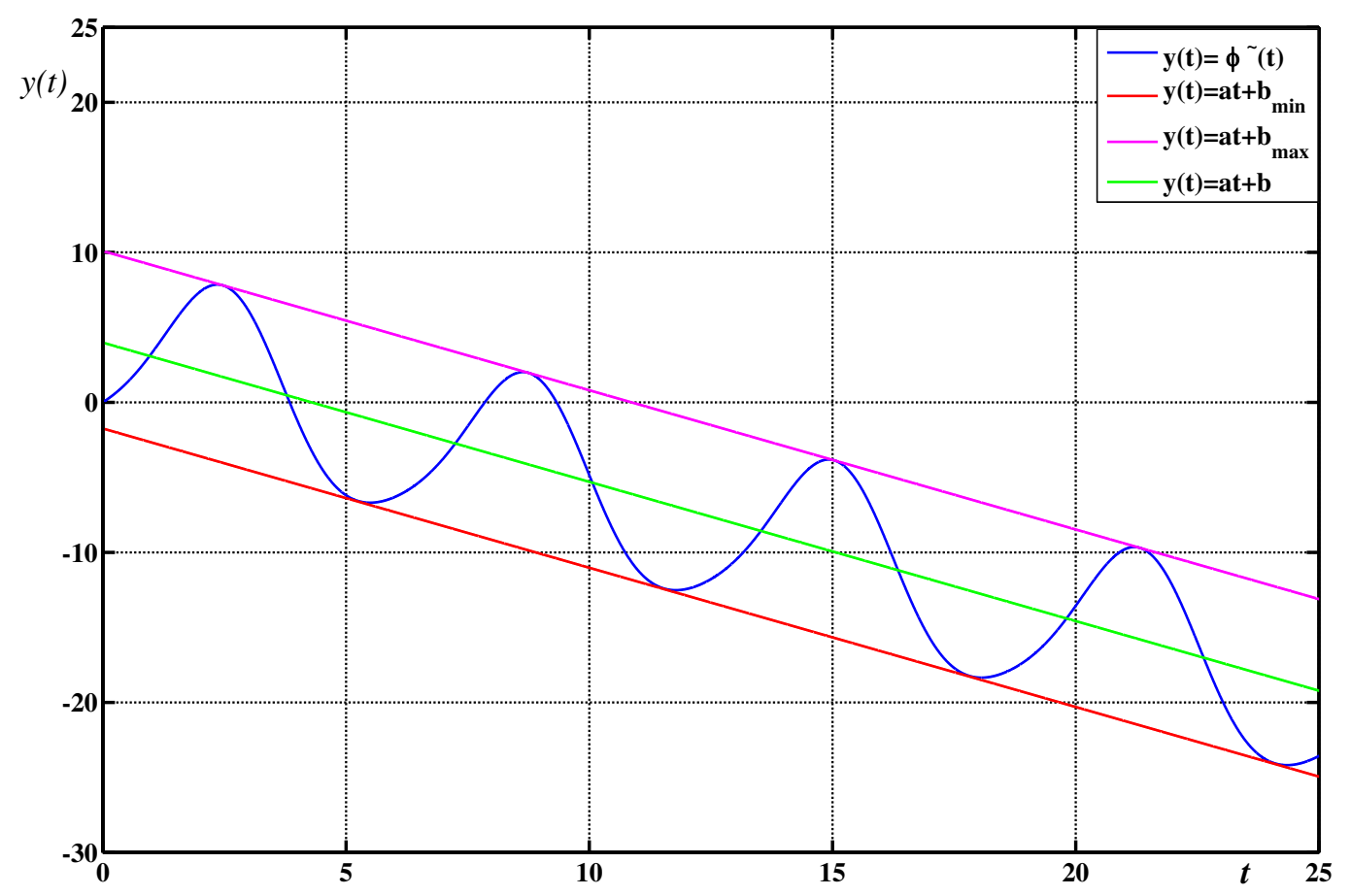

Fig. 19. The strip of oscillation of $\tilde{\varphi}(t)$ bounded by the lines $T_{\min }$ and $T_{\max }$ for $\alpha_{1}=1$ and $\alpha_{2}=0.5$.

which is tangent of $\gamma_{\tilde{\varphi}}$ in these minima, and its local maxima lie in the straight line

$$
T_{\max }: y=A t+B_{\max },
$$

which is tangent of $\gamma_{\tilde{\varphi}}$ in these maxima.
Figure 20 illustrates the evolution of the slope of $T_{\min }$ and $T_{\max }$ for $0 \leq \alpha_{2} \leq 1$. We can calculate $B_{\min }$ and $B_{\max }$ as follows

$$
B_{\min }=\tilde{\varphi}\left(t_{\min }\right)-A t_{\min }
$$

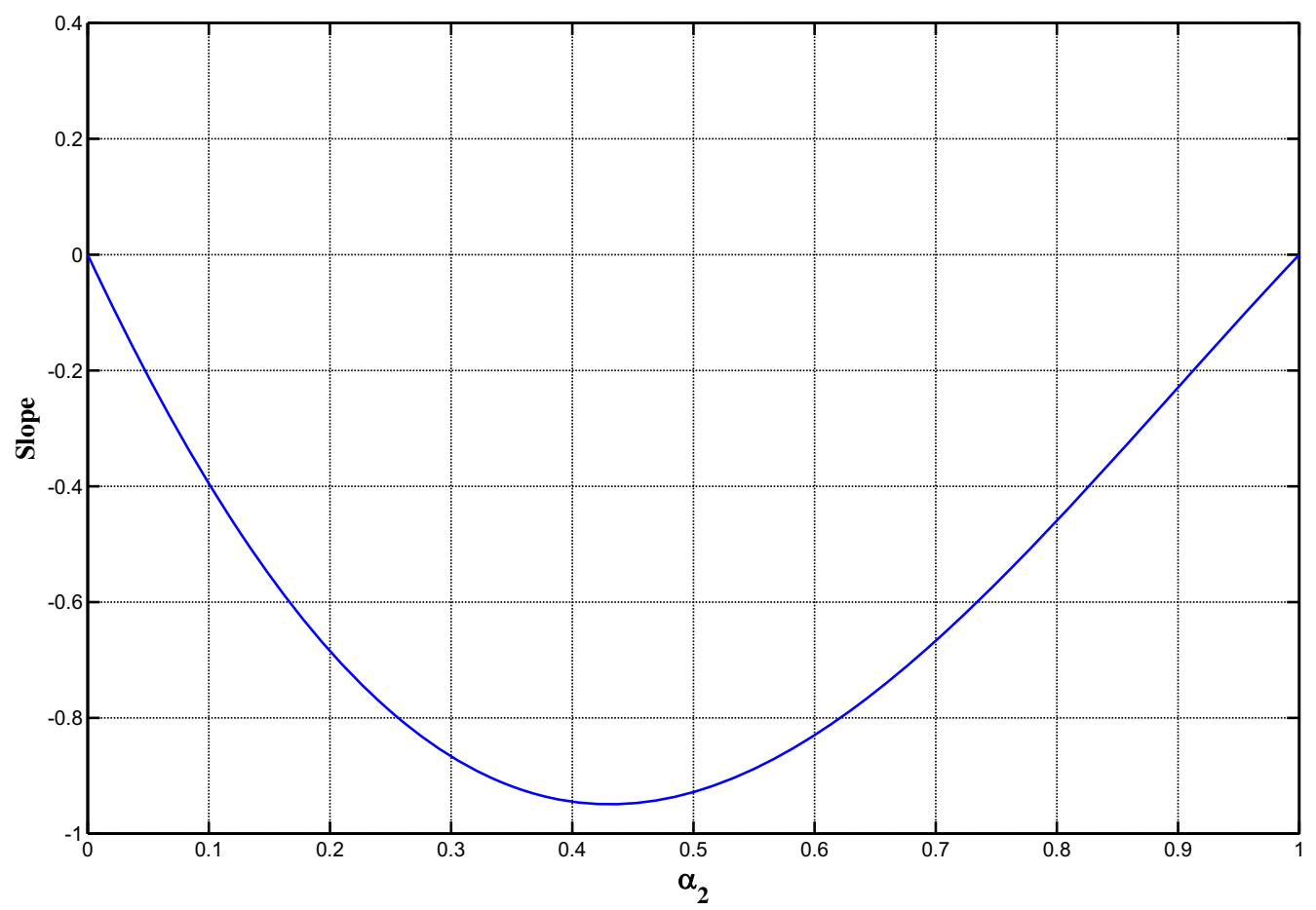

Fig. 20. Variation of the slope of the strip containing the flux oscillations for $\alpha_{1}=1$ and $0 \leq \alpha_{2} \leq 1$. 
and

$$
B_{\max }=\tilde{\varphi}\left(t_{\max }\right)-A t_{\max }
$$

where $t_{\min }, t_{\max }$ are the abscissas of the extrema and can be calculated solving the equation

$$
\tilde{v}(t)=A,
$$

or equivalently

$$
P(t)=0 .
$$

It is not easy to give the closed formula (function of $\alpha_{2}$ ) for the solutions of this equation, so we are only able to give numerical solutions for some values of $\alpha_{2}$, the results are illustrated in Table 3 .

In order to give a general formula for $B_{\min }$, $B_{\max }$ as functions of $\alpha_{2}$ we use the fourth degree Lagrange polynomial interpolation based on the value of $B_{\min }, B_{\max }$ calculated for five values of $\alpha_{2}$ $\left(\alpha_{2}=0,0.2,0.5,0.8\right.$ and 1$)$ then we obtain the following approximate expressions for $0 \leq \alpha_{2} \leq 1$

$$
\begin{aligned}
B_{\min } \approx & -6.88 \alpha_{2}^{4}+22.43 \alpha_{2}^{3}-16.46 \alpha_{2}^{2} \\
& +0.08 \alpha_{2}, \\
B_{\max } \approx & -1.45 \alpha_{2}^{4}+11.18 \alpha_{2}^{3}-9.60 \alpha_{2}^{2} \\
& -18.97 \alpha_{2}+20.66 .
\end{aligned}
$$

Proposition 3. The graph $\gamma_{\tilde{\varphi}}$ of $\tilde{\varphi}$ lies in a strip of width

$$
\tilde{L}=\left(B_{\max }-B_{\min }\right) \cos (\arctan (A)) .
$$

Proof. Setting $Q\left(-\frac{B_{\max }}{A}, 0\right)$ as the intersection between $T_{\max }$ and the axe of abscissas, $R\left(-\frac{B_{\max }}{A},-B_{\max }+B_{\min }\right)$ the point in $T_{\min }$ of abscissas $-\frac{B_{\max }}{A}$ and $S$ the intersection between the

Table 3. Numerical solutions of Eq. (61) for some values of $\alpha_{2}($ with $k \in \mathbf{N})$.

\begin{tabular}{lcccr}
\hline$\alpha_{2}$ & $t_{\min }$ & $B_{\min }$ & $t_{\max }$ & $B_{\max }$ \\
\hline 0 & $2 \pi k$ & 0 & $2 \pi(k+1)$ & 20.67 \\
0.1 & $2 \pi k-0.24$ & -0.14 & $2 \pi k+3.01$ & 18.68 \\
0.2 & $2 \pi k-0.47$ & -0.47 & $2 \pi k+2.88$ & 16.58 \\
0.3 & $2 \pi k-0.67$ & -0.91 & $2 \pi k+2.74$ & 14.40 \\
0.4 & $2 \pi k-0.86$ & -1.34 & $2 \pi k+2.61$ & 12.22 \\
0.5 & $2 \pi k-1.03$ & -1.70 & $2 \pi k+2.47$ & 10.09 \\
0.6 & $2 \pi k-1.18$ & -1.93 & $2 \pi k+2.32$ & 8.06 \\
0.7 & $2 \pi k-1.31$ & -1.97 & $2 \pi k+2.16$ & 6.17 \\
0.8 & $2 \pi k-1.44$ & -1.81 & $2 \pi k+1.99$ & 4.48 \\
0.9 & $2 \pi k-1.54$ & -1.43 & $2 \pi k+1.79$ & 3.02 \\
1 & $2 \pi k-\frac{\pi}{2}$ & -0.83 & $2 \pi k+\frac{\pi}{2}$ & 1.83 \\
\hline
\end{tabular}

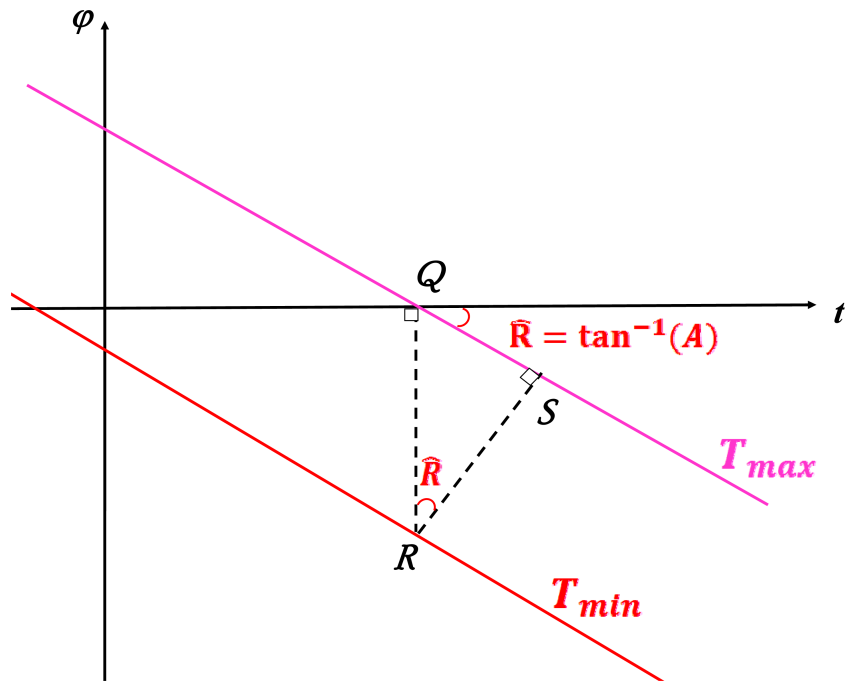

Fig. 21. Orthogonal distance between the lines bounding the oscillation of $\tilde{\varphi}(t)$ for $\alpha_{1}=1$ and $0 \leq \alpha_{2} \leq 1$.

orthogonal to $T_{\min }$ at $R$ and $T_{\max }$. The triangle QRS will be orthogonal at $S$ and $R S$ will be the orthogonal distance between $T_{\max }$ and $T_{\min }$. We have

$$
\hat{R}=|\arctan (A)|
$$

and

$$
\cos (\hat{R})=\frac{R S}{Q R}
$$

then

$$
\begin{aligned}
\tilde{L} & =R S=Q R \cos (\hat{R}) \\
& =\left(B_{\max }-B_{\min }\right) \cos (\arctan (A)) .
\end{aligned}
$$

Proposition 4. The evolution of $\varphi$ is practically similar to that of $\tilde{\varphi}(t)$ and the graph $\gamma_{\varphi}$ of $\varphi$ lies in a strip of a bounded width $L$ which converges to

$$
\tilde{L}=\left(B_{\max }-B_{\min }\right) \cos (\arctan (A)) .
$$

Proof. Integrating both sides of (54) gives

$$
\begin{aligned}
\varphi(t) & =A t+\int_{0}^{t} P(\tau) d \tau+\int_{0}^{t} \xi(\tau) d \tau \\
& =\tilde{\varphi}(t)+\int_{0}^{t} \xi(\tau) d \tau,
\end{aligned}
$$

adding $\int_{0}^{t} \xi(\tau) d \tau$ to both sides of (58) yields

$$
\begin{aligned}
A t+ & B_{\min }+\int_{0}^{t} \xi(\tau) d \tau \\
& \leq \varphi(t) \leq A t+B_{\max }+\int_{0}^{t} \xi(\tau) d \tau
\end{aligned}
$$


this means that the graph of $\varphi(t)$ oscillates in a strip bounded by the lower curve $\Gamma_{\min }$ of equation

$$
y_{\min }(t)=A t+B_{\min }+\int_{0}^{t} \xi(\tau) d \tau
$$

and the upper curve $\Gamma_{\max }$ of equation

$$
y_{\max }(t)=A t+B_{\max }+\int_{0}^{t} \xi(\tau) d \tau
$$

differentiating both two equations yields

$$
\frac{d y_{\min }(t)}{d t}=\frac{d y_{\max }(t)}{d t}=A+\xi(t)
$$

we have $\xi(t) \rightarrow 0$ for $t \gg 0$ so the slopes of the tangents at $\Gamma_{\min }$ and at $\Gamma_{\max }$ converge to the constant $A$, this means that $\Gamma_{\min }$ and $\Gamma_{\max }$ converges towards two straight lines. On the other hand, the distance between the point $Q\left(t, y_{\min }(t)\right)$ of $\Gamma_{\min }$ and the point $R\left(t, y_{\max }(t)\right)$ is

$$
Q R=y_{\max }(t)-y_{\min }(t)=B_{\max }-B_{\min }
$$

then with similar procedure to the proof of Proposition 3, we can show that the orthogonal distance $L$ between $\Gamma_{\min }$ and $\Gamma_{\max }$ converges to

$$
\tilde{L}=\left(B_{\max }-B_{\min }\right) \cos (\arctan (A)) .
$$

Figure 22 illustrates the band of $\varphi$ compared with the band of $\tilde{\varphi}$ for $\alpha_{1}=1$ and $\alpha_{2}=0.5$.

\subsection{Behavior of memfractance for miscellaneous values of $\alpha_{1}, \alpha_{2}$}

In this subsection, we illustrate the interpolated characteristic of a memfractor between a memristor, a memcapacitor, a meminductor and a second-order memristor, by varying both $\alpha_{1}$ and $\alpha_{2}$ simultaneously with $\left(0 \leq \alpha_{1} \leq 1,0 \leq \alpha_{2} \leq\right.$ $1)$. Figure 23 displays the time variation of voltage $(v-t$ curve) which has a periodic waveform in any case.

Figure 24 shows the hysteresis loop (Lissajous curve) in the $v-i$ plane for $\left(0 \leq \alpha_{1} \leq 1\right.$ and $0 \leq \alpha_{2} \leq 1$ ), when $\alpha_{1}=\alpha_{2}=0$ (second-order memristor case) the loop collapses to a single-valued function, in this case we have

$$
\begin{aligned}
\varphi(t) & =\left(1+\sigma(t)+\sigma^{2}(t)\right) q(t) \\
& =\left(1+(1-\cos (t))+(1-\cos (t))^{2}\right) \sin (t) \\
& =\cos ^{2}(t) \sin (t)-3 \cos (t) \sin (t)+3 \sin (t),
\end{aligned}
$$

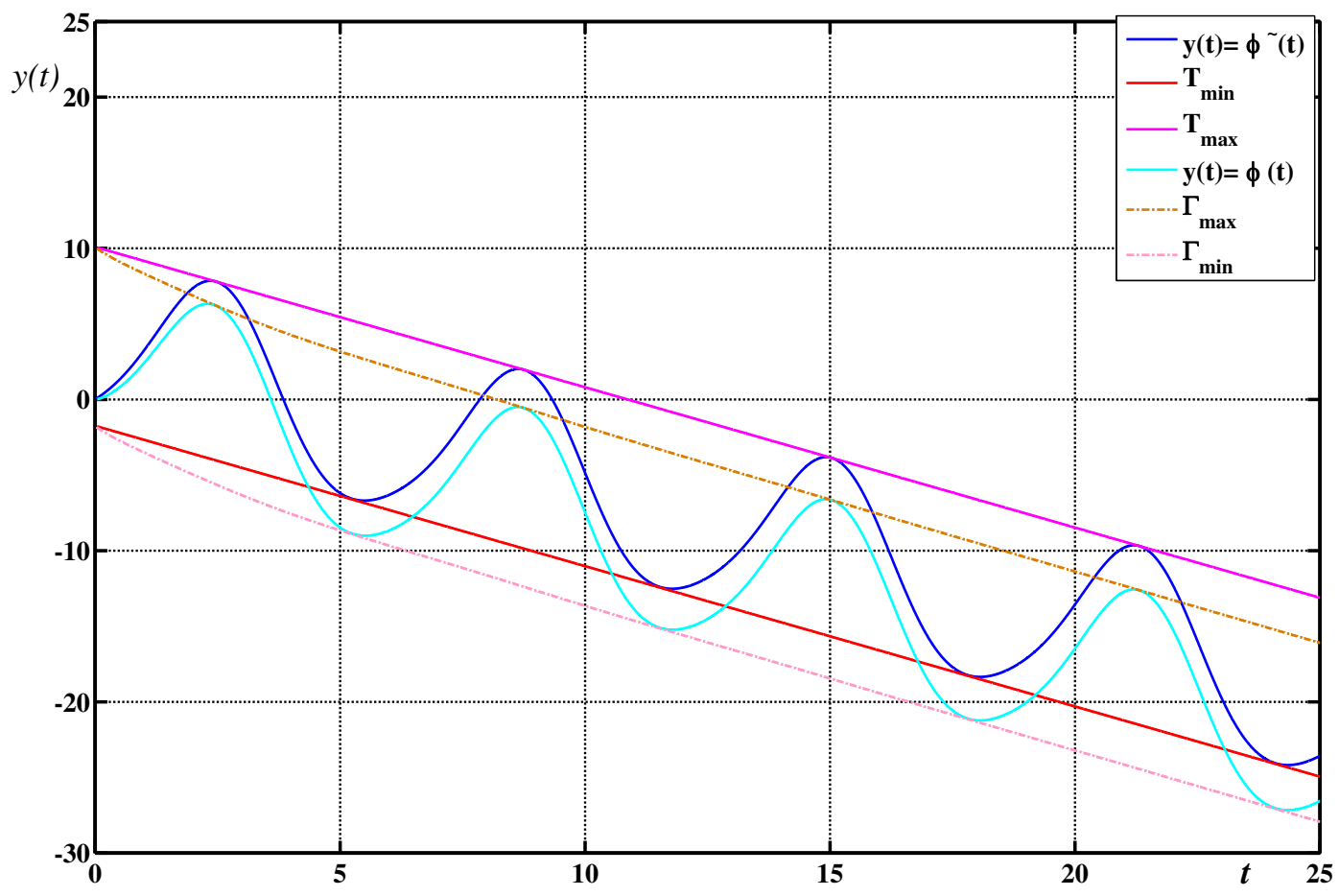

Fig. 22. The band of oscillation of $\varphi(t)$ bounded by the curves $\Gamma_{\min }: y(t)=a t+b_{\min }+\int_{0}^{t} \xi(\tau) d \tau$ and $\Gamma_{\max }: y(t)=$ $a t+b_{\max }+\int_{0}^{t} \xi(\tau) d \tau$, for $\alpha_{1}=1$ and $\alpha_{2}=0.5$. 


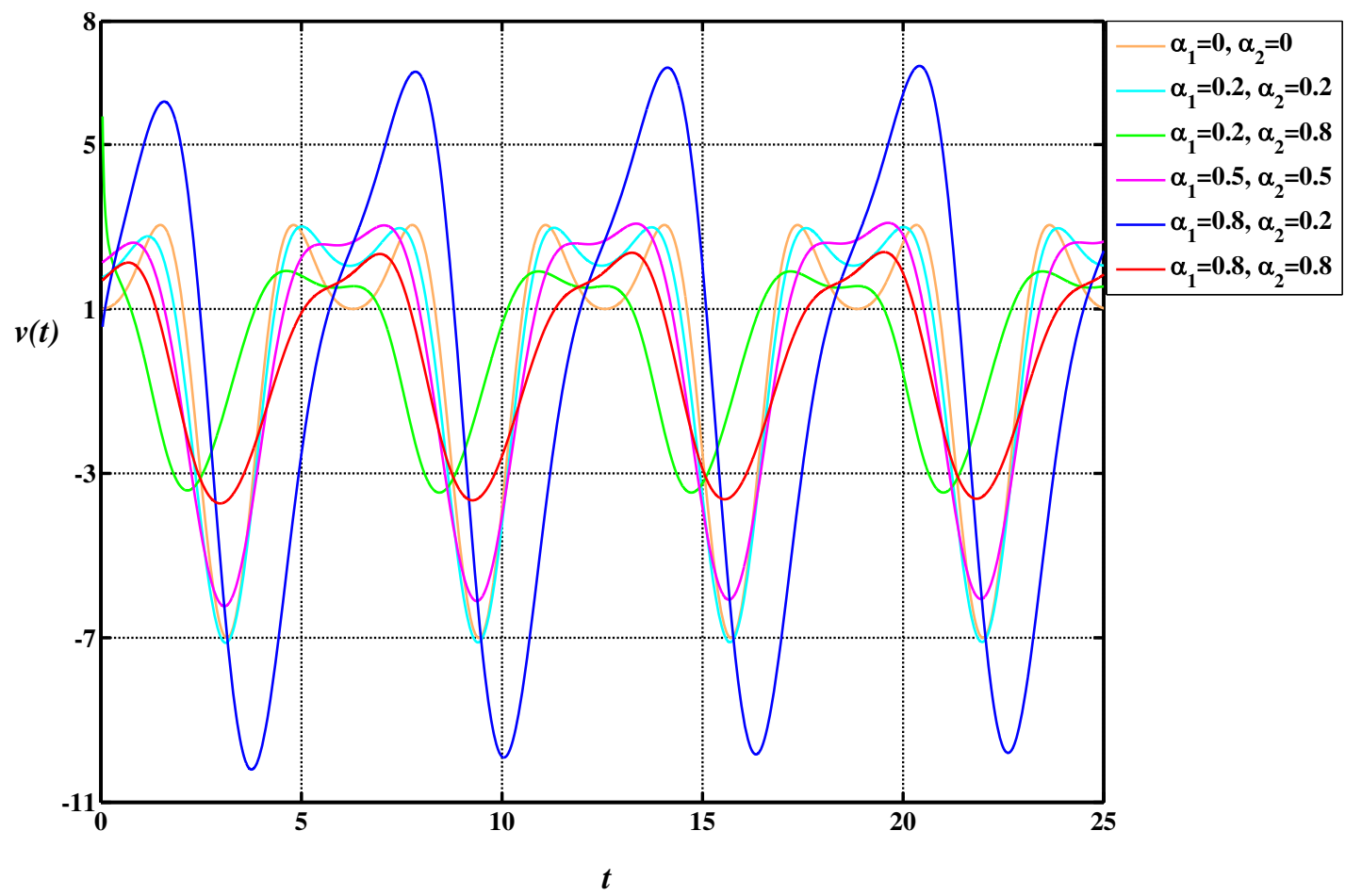

Fig. 23. Periodic waveform of $\left(v-t\right.$ curve) for $\left(0 \leq \alpha_{1} \leq 1,0 \leq \alpha_{2} \leq 1\right.$ obtained by solving $(33)$ with $F_{M}^{\alpha_{1}, \alpha_{2}}(t)=$ $\alpha_{1}\left(1-\alpha_{2}\right)\left(1+(3-\cos (t))^{2}\right)+\alpha_{1} \alpha_{2}\left(\frac{\alpha_{1}+\alpha_{2}}{2}\right)\left(1+\sin (t)+\sin ^{2}(t)\right)+\alpha_{2}\left(1-\alpha_{1}\right)\left(1+e^{\sin (t)}\right)+\left(1-\alpha_{1}\right)\left(1-\alpha_{2}\right)(1+(1-\cos (t))+$ $\left.\sin ^{2}(t)\right)$.

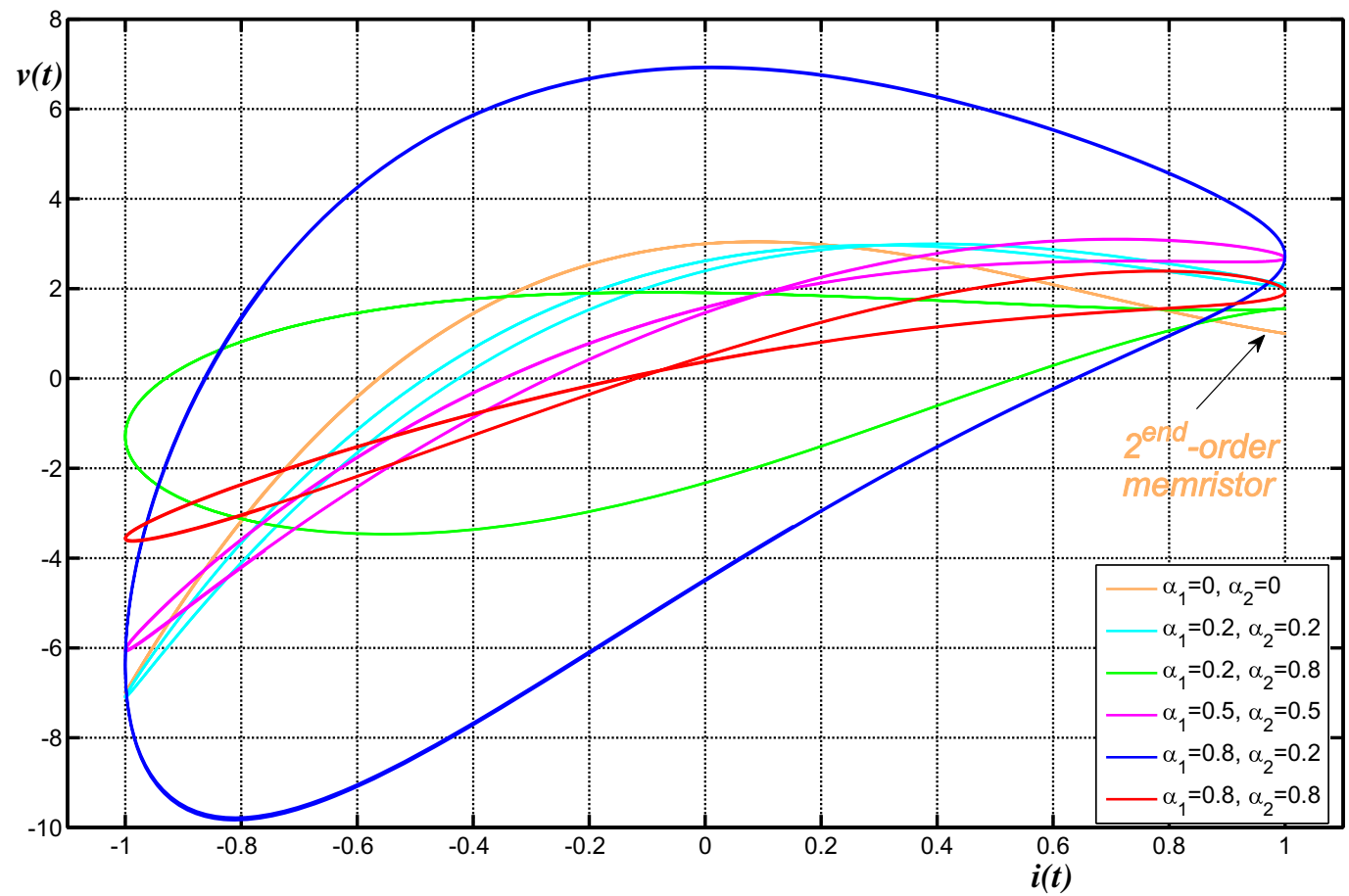

Fig. 24. Hysteresis loop (Lissajous curve) associated with Fig. 23 (Example 5.3) in the $v-i$ plane for $\left(0 \leq \alpha_{1} \leq 1,0 \leq \alpha_{2} \leq 1\right)$. 


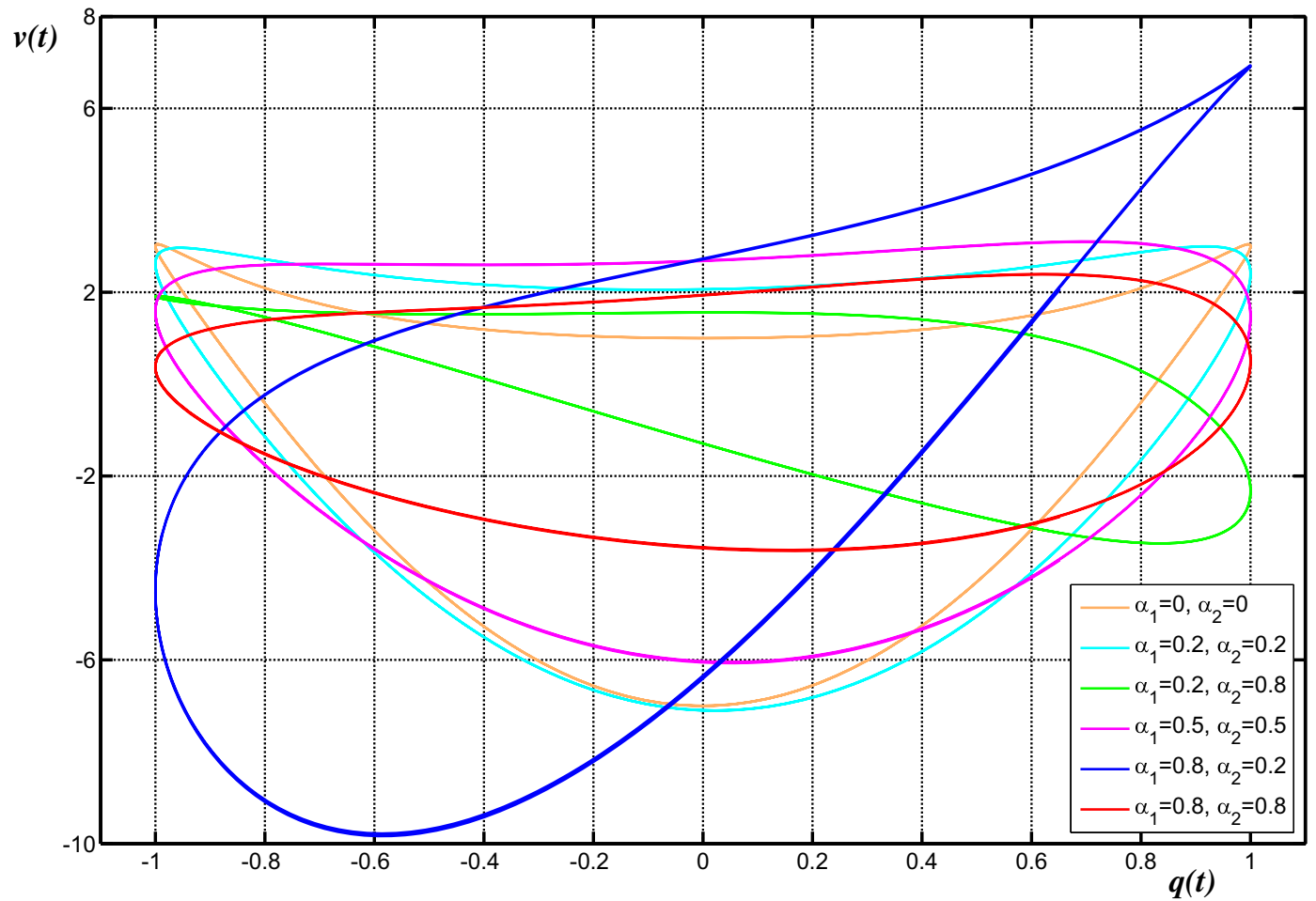

Fig. 25. Hysteresis loop (Lissajous curve) associated with Fig. 23 (Example 5.3) in the $v-q$ plane for $\left(0 \leq \alpha_{1} \leq 1,0 \leq \alpha_{2} \leq 1\right.$ and $\left.\alpha_{1}+\alpha_{2} \geq 1\right)$.

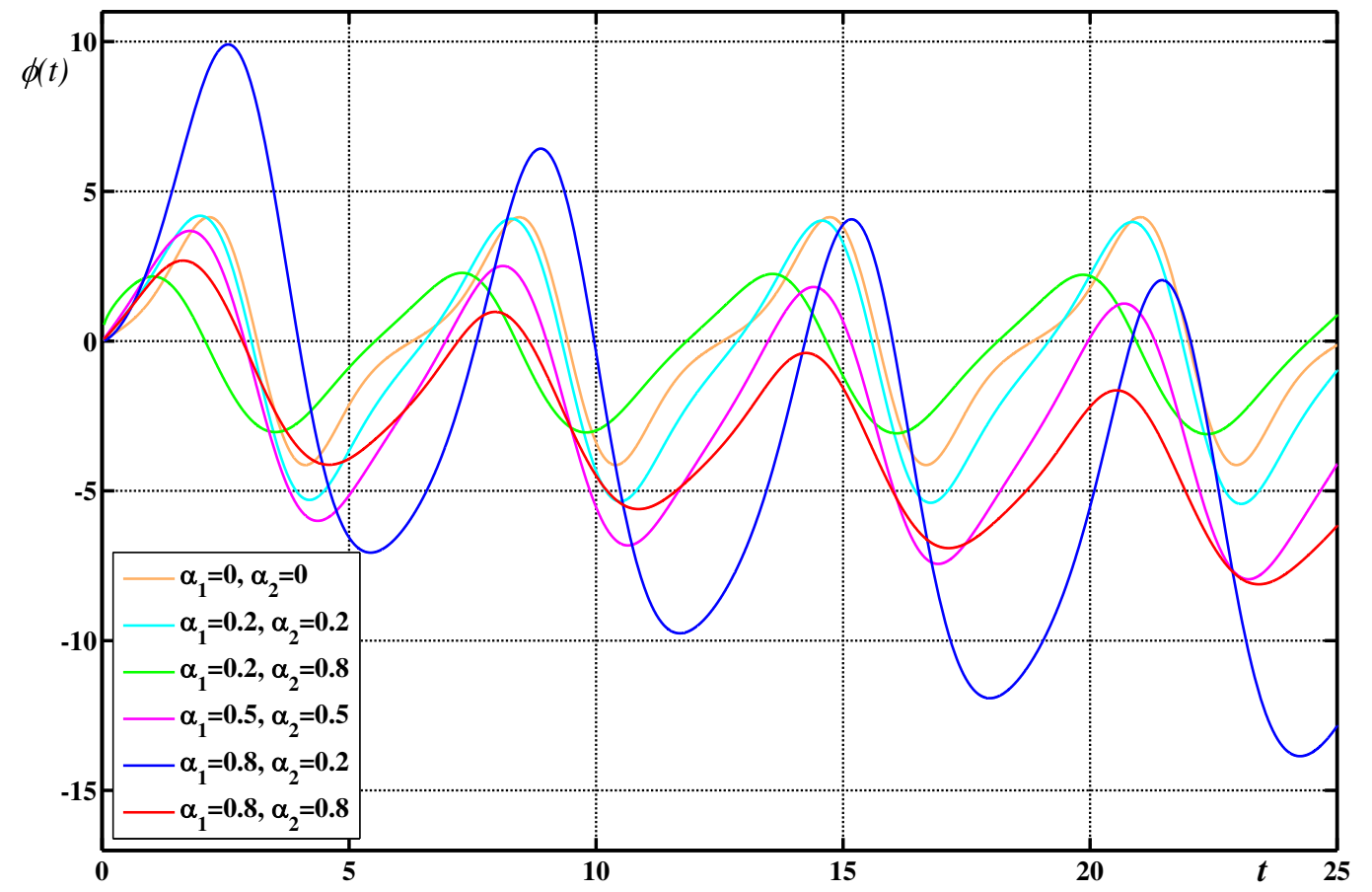

Fig. 26. Oscillation-form of ( $\varphi-t$ curve) associated with Fig. 23 (Example 5.3) for $\left(0 \leq \alpha_{1} \leq 1,0 \leq \alpha_{2} \leq 1\right)$. 
differentiating (66) with respect to $t$ gives

$$
\begin{aligned}
v(t) & =3 \cos ^{3}(t)-6 \cos ^{2}(t)+\cos (t)+3 \\
& =3 i^{3}(t)-6 i^{2}(t)+i(t)+3
\end{aligned}
$$

which is a single valued function of $i$. Upon varying $\alpha_{1}$ and $\alpha_{2}$, various forms of the loop are observed.

Figure 25 shows the hysteresis loop (Lissajous curve) in the $v-q$ plane for $\left(0 \leq \alpha_{1} \leq 1,0 \leq\right.$ $\alpha_{2} \leq 1$ ), when $\alpha_{1}=\alpha_{2}=0$ (second-order memristor case) the hysteresis loop is a double-valued Lissajous curve in this case, we have

$$
\begin{aligned}
v(t)= & 6 \sin ^{2}(t) \\
& -3 \pm\left(4-3 \sin ^{2}(t)\right) \sqrt{1-\sin ^{2}(t)} \\
= & 6 q^{2}(t)-3 \pm\left(4-3 q^{2}(t)\right) \sqrt{1-q^{2}(t)},
\end{aligned}
$$

which is a double-valued function of $q$.

Figure 26 displays the time variation of flux $\left(\varphi-t\right.$ curve) for $\left(0 \leq \alpha_{1} \leq 1,0 \leq \alpha_{2} \leq 1\right)$ which has an oscillation form in a horizontal band for $\left(\alpha_{1}=0\right.$ or $\alpha_{2}=1$ ) but the band of oscillation inclines in the other cases.

These three subsections affirm the fact that any state between meminductor, memristor, memcapacitor and second-order memristor can be modulated by a generalized memfractor when choosing a convenient fractional derivative of orders $\alpha_{1}$ and $\alpha_{2}$ such that $\left(0 \leq \alpha_{1} \leq 1,0 \leq \alpha_{2} \leq 1\right)$.

\section{Extending the Generalized \\ Ohm's Law to the Periodic Table of Circuit Elements}

In this section, we extend the generalized Ohm's law (36) in order to embed the memfractor element in the periodic table of circuit elements defined by Fig. 27 and to link the symbols of local variables $\left(\alpha_{1}, \alpha_{2}\right)$ belonging to $[0,1] \times[0,1]$ to the symbols of the global variables $\alpha$ and $\beta$ which are standard symbols in the circuit theory community. Chua [2012] has introduced the notion of $(\alpha, \beta)$ elements which defines an infinite discrete family of circuit elements, each one identified by its element code $\left(v^{(\alpha)}, i^{(\beta)}\right)$, where $|\alpha|,|\beta|$ are integers, and the symbol $v^{(\alpha)}$ (the symbol $i^{(\beta)}$ respectively) means the $\alpha$ th derivative of the voltage $v$ (the $\beta$ th derivative of the current $i$ respectively) if $\alpha \geq 0$ ( $\beta \geq 0$ respectively) and the $|\alpha|$ th time integral of the voltage $v$ (the $|\beta|$ th time integral of the current $i$, respectively) if $\alpha<0$ ( $\beta<0$ respectively).
This infinite family of circuit elements is essential for developing a rigorous mathematical theory of nonlinear circuits and is not defined for the sake of generality.

\subsection{General Ohm's law for electric circuit elements}

The term integer circuit elements is used henceforth as a moniker for the circuit elements identified as an integer pair $(\alpha, \beta)$ elements. The three classical elements resistor, capacitor and inductor are identified as the $\left(v^{(0)}, i^{(0)}\right),\left(v^{(0)}, i^{(-1)}\right)$ and $\left(v^{(-1)}, i^{(0)}\right)$ elements respectively for which we have the relations $v=R i, v=\frac{1}{C} q$ and $\varphi=L i$, respectively or equivalently

$$
\begin{aligned}
d v & =R d i, \\
d v & =\frac{1}{C} d q, \\
d \varphi & =L d i .
\end{aligned}
$$

Using the code element $\left(v^{(\alpha)}, i^{(\beta)}\right)$ and taking into account that

$$
\left\{\begin{array}{l}
v=\frac{d \varphi}{d t} \\
i=\frac{d q}{d t}
\end{array}\right.
$$

Eqs. (70)-(72) can be represented by a single equation, namely

$$
d v^{(\alpha)}=M^{\alpha, \beta} d i^{(\beta)},
$$

where

$$
M^{\alpha, \beta}= \begin{cases}R, & \text { if } \alpha=\beta=0, \\ \frac{1}{C}, & \text { if } \alpha=0, \beta=-1, \\ L, & \text { if } \alpha=-1, \beta=0 .\end{cases}
$$

The three circuit elements with memory memristor, memcapacitor and meminductor (bottom square of Fig. 4) are identified as the $\left(v^{(-1)}, i^{(-1)}\right)$, $\left(v^{(-1)}, i^{(-2)}\right)$ and $\left(v^{(-2)}, i^{(-1)}\right)$ elements respectively for which we have the relations

$$
\begin{aligned}
& d \varphi=R_{M} d q, \\
& d \varphi=C_{M}^{-1} d \sigma, \\
& d \rho=L_{M} d q,
\end{aligned}
$$

respectively. 


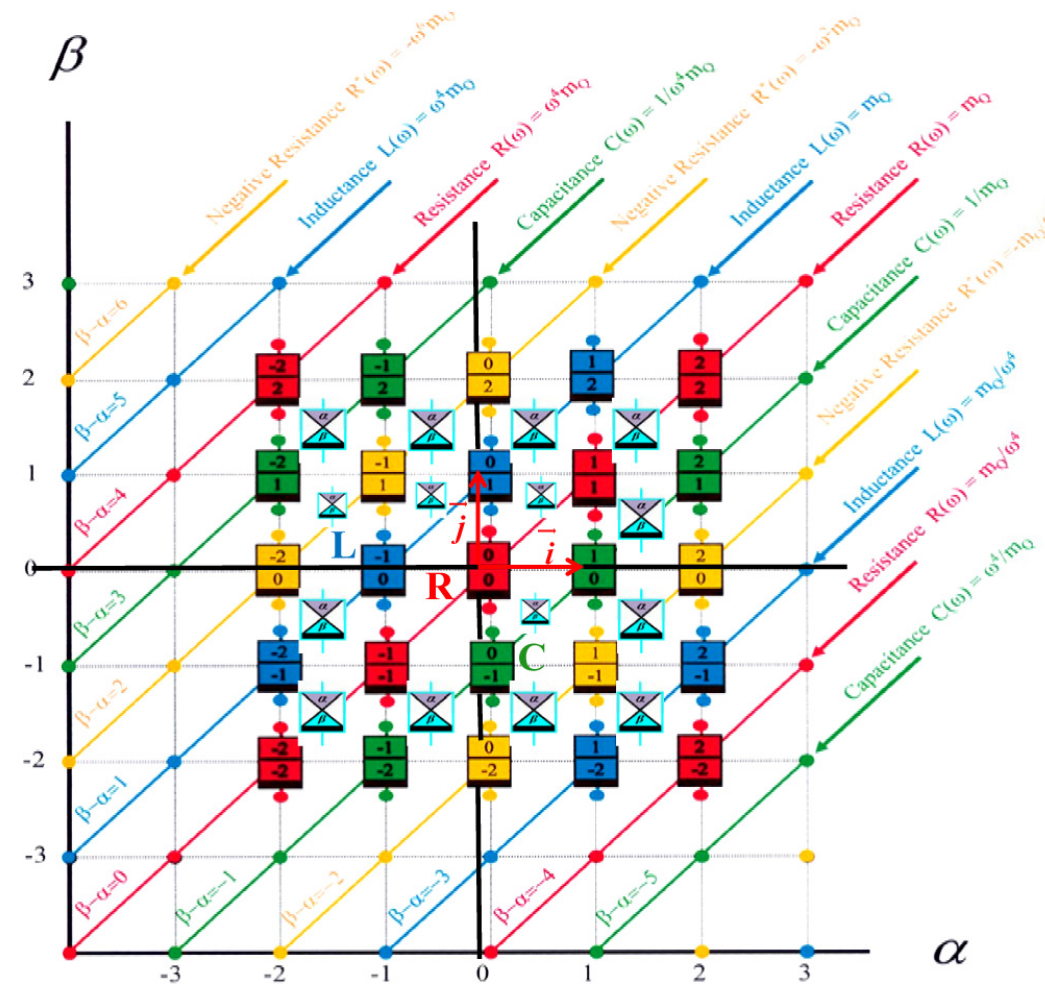

Fig. 27. Periodic table of circuit elements embedded in the $\mathbb{R}^{2}$ plane.

Using the code element $\left(v^{(\alpha)}, i^{(\beta)}\right)$, these equations can be represented by the single Eq. (74) where

$$
M^{\alpha, \beta}= \begin{cases}R_{M}, & \text { if } \alpha=\beta=-1, \\ C_{M}^{-1}, & \text { if } \alpha=-1, \beta=-2, \\ L_{M}, & \text { if } \alpha=-2, \beta=-1 .\end{cases}
$$

The three circuit elements with second-order memory: second-order memristor, second-order memcapacitor, second-order meminductor and the circuit element with third-order memory: thirdorder memristor (upper square of Fig. 4) are identified as the $\left(v^{(-2)}, i^{(-2)}\right),\left(v^{(-2)}, i^{(-3)}\right),\left(v^{(-3)}, i^{(-2)}\right)$ and $\left(v^{(-3)}, i^{(-3)}\right)$ elements respectively for which we give the relations

$$
\begin{aligned}
d \rho & =R_{2 M} d \sigma, \\
d \rho & =C_{M}^{-1} d \sigma_{1}, \\
d \rho_{1} & =L_{M} d \sigma, \\
d \rho_{1} & =R_{3 M} d \sigma_{1} .
\end{aligned}
$$

Using again the code element $\left(v^{(\alpha)}, i^{(\beta)}\right)$, these equations can be represented by the single Eq. (74) where

$$
M^{\alpha, \beta}= \begin{cases}R_{2 M}, & \text { if } \alpha=\beta=-2, \\ C_{2 M}^{-1}, & \text { if } \alpha=-2, \beta=-3, \\ L_{2 M}, & \text { if } \alpha=-3, \beta=-2, \\ R_{3 M}, & \text { if } \alpha=\beta=-3 .\end{cases}
$$

Multiplying both sides of (74) by $\frac{1}{d t}$ yields

$$
\frac{d}{d t} v^{(\alpha)}=M^{\alpha, \beta} \frac{d}{d t} i^{(\beta)},
$$

which is equivalent to

$$
v^{(\alpha+1)}=M^{\alpha, \beta} i^{(\beta+1)}
$$

and taking into account the relations (73) we obtain

$$
\varphi^{(\alpha+2)}=M^{\alpha, \beta} q^{(\beta+2)} .
$$

Setting

$$
\left\{\begin{array}{l}
\alpha_{1}=\alpha+1, \\
\alpha_{2}=\beta+1, \\
F^{\alpha_{1}, \alpha_{2}}=M^{\alpha, \beta},
\end{array}\right.
$$

we recover our generalized constitutive relations (20) and (21), where in this case $\alpha_{1}, \alpha_{2}$ are integer numbers for which the fractional derivative becomes a conventional derivative. 
In the same manner, setting

$$
\left\{\begin{array}{l}
\alpha_{1}=\alpha+2, \\
\alpha_{2}=\beta+2, \\
F_{M}^{\alpha_{1}, \alpha_{2}}=M^{\alpha, \beta},
\end{array}\right.
$$

we recover (36) and (32).

\subsection{Extending generalized Ohm's law}

If we assume $\alpha_{1}=\alpha+2, \alpha_{2}=\beta+2$ belonging in $\mathbb{R}$, then the relation (74) (in term of conventional derivatives) which corresponds to the integers values of $\alpha_{1}, \alpha_{2}$ becomes a special case of (32) (in terms of fractional derivatives which are a generalization of the conventional derivatives).

Replacing $\left(\alpha_{1}, \alpha_{2}\right)$ in $(32)$ by $(\alpha+2, \beta+2)$ and $F_{M}^{\alpha_{1}, \alpha_{2}}$ by $M^{\alpha, \beta}$ yields

$$
D_{t}^{\alpha+2} \varphi(t)=M^{\alpha, \beta}(t) D_{t}^{\beta+2} q(t),
$$

taking into account the relations (12) (with assumptions $t_{0}=0, q\left(t_{0}\right)=0$ and $\varphi\left(t_{0}\right)=0$ ) and the relation (5), Eq. (87) can be written as follows

$$
D_{t}^{\alpha+1} v(t)=M^{\alpha, \beta}(t) D_{t}^{\beta+1} i(t),
$$

taking into account the relation (6) we obtain

$$
\frac{d}{d t}\left(D_{t}^{\alpha} v(t)\right)=M^{\alpha, \beta}(t) \frac{d}{d t}\left(D_{t}^{\beta} i(t)\right) .
$$

Multiplying both sides of (89) by $d t$ yields

$$
d\left(D_{t}^{\alpha} v(t)\right)=M^{\alpha, \beta}(t) d\left(D_{t}^{\beta} i(t)\right) .
$$

Remark 6.1. In the same way replacing $\left(\alpha_{1}, \alpha_{2}\right)$ in (21) by $(\alpha+1, \beta+1)$ and $F_{M}^{\alpha_{1}, \alpha_{2}}$ by $M^{\alpha, \beta}$ we obtain the same equations (87)-(90).

Using the enlargement of notation code $(\alpha, \beta)$ to real values of $\alpha, \beta$, we define an infinite continued family of circuit elements. We call this family: fractional circuit elements family. Hence the periodic table of circuit elements introduced by Chua [2012] is embedded in the Euclidean plane $\mathbb{R}^{2}$ with the standard orthonormal basis $(\vec{i}, \vec{j})$ where $\vec{i}=-\overrightarrow{R L}, \vec{j}=-\overrightarrow{R C}$, the origin $R(0,0)$ is the resistor, $C(0,-1)$ is the capacitor and $L(-1,0)$ is the inductor (Fig. 27). In this representation, each element $(\alpha, \beta)$ can be viewed as a point of coordinate $(\alpha, \beta)$ and therefore can be written as $(\alpha, \beta)=$ $\alpha \vec{i}+\beta \vec{j}=-(\alpha \overrightarrow{R L}+\beta \overrightarrow{R C})$ where the abscissa $\alpha$ indicates the dynamic order of the element in the direction of inductance and the ordinate $\beta$ indicates the dynamic order of the element in the direction of capacitance. When $\alpha=\beta$ the element $(\alpha, \beta)$ behaves in a certain way like a resistance. We can observe (Fig. 24) that for $\alpha_{1}=\alpha_{2}=0$ (i.e. $\alpha=\beta=-2$ ), $\alpha_{1}=\alpha_{2}=0.2$ (i.e. $\alpha=\beta=-1.8$ ), $\alpha_{1}=\alpha_{2}=0.5$ (i.e. $\alpha=\beta=-1.5$ ) and $\alpha_{1}=\alpha_{2}=$ 0.8 (i.e. $\alpha=\beta=-1.2)$ the dynamic of the $(\alpha, \beta)$ element is similar to that of the resistor, furthermore its hysteresis loop (Lissajous curve) in the $v-i$ plane is punched or encapsulated on a single-valued function but for $\alpha_{1}=0.2, \alpha_{2}=0.8$ (i.e. $\alpha=-1.8$, $\beta=-1.2$ ) and $\alpha_{1}=0.8, \alpha_{2}=0.2$ (i.e. $\alpha=-1.2$, $\beta=-1.8$ ) its dynamic is completely different from that of the resistor.

Remark 6.2. The Euclidean distance of any fractional element $M^{\alpha, \beta}$ from the resistor which is the origin is

$$
d\left(R, M^{\alpha, \beta}\right)=\left\|\overrightarrow{R M^{\alpha, \beta}}\right\|=\sqrt{\alpha^{2}+\beta^{2}} .
$$

Using the complexity metric $\chi$ [Chua, 2012] the distance of the element $M^{\alpha, \beta}$ from the resistor is

$$
d\left(R, M^{\alpha, \beta}\right)=\chi(\alpha, \beta)=|\alpha|+|\beta|,
$$

the second distance may be more useful than the first one because in the integer case the complexity metric measures not just the distance of $(\alpha, \beta)$ element from the resistor, but also the minimum number of capacitors (or inductors) needed to build an $(\alpha, \beta)$ element using off-the-shelf components.

Remark 6.3. For a given pair $(\alpha, \beta)$ the corresponding fractional circuit element $M^{\alpha, \beta}$ is localized inside of the square formed by the four integer circuit elements $(E(\alpha), E(\beta)),(E(\alpha)+1, E(\beta))$, $(E(\alpha), E(\beta)+1)$ and $(E(\alpha)+1, E(\beta)+1)$, where for any real $x$, the function $E(x)$ denotes the integer part of $x$.

\section{Conclusion}

In this paper, we have used fractional calculus in order to generalize and provide a mathematical frame for circuit elements with memory: memfractance. We have emphasized that the memfractance is a general paradigm for unifying and enlarging the family of memristive, memcapacitive and meminductive elements. The motivation and significance of this paper is that there may exist future nanoelectronics devices that are more realistically modeled with memfractance elements. 
We have generalized the definition of fractance which was first introduced in 1983, and after that, introduced the paradigm of memfractance which is fitted for circuit elements with memory such as memristor, meminductor, memcapacitor and second-order memristor first introduced here. We have defined a new element called memfractor which possesses interpolated characteristics between those four circuit elements.

We have then generalized Ohm's law to memfractor and proved it. A particular, albeit wideranging, case of memfractance: the interpolated memfractance has been carefully studied through several numerical illustrative examples. Special attention has been devoted to the interpolated characteristic of a memfractor lying between memristor and memcapacitor which exhibits an unexpected new behavior of time variation of flux ( $\varphi-t$ curve). This phenomenon has been studied very carefully by the means of rigorous proofs.

Finally, following Chua's recent work in which an infinite discrete family of circuit elements: the $(\alpha, \beta)$ element is introduced in the scope of developing a rigorous mathematical theory of nonlinear circuits, we extend the previous generalized Ohm's law in order to embed memfractors elements into this periodic table. For this aim, we define an infinite continued family of circuit elements including circuit elements with memory (such as second-order memcapacitor and meminductor and third-order memristor), with a special metric. We call this family: fractional circuit element family.

\section{Acknowledgment}

This paper is supported in part by AFOSR grant number FA9550-13-1-0136 and the Marie-Curie Fellowship.

\section{References}

Bagley, R. L. \& Calico, R. A. [1991] "Fractional order state equations for the control of viscoelastically damped structures," J. Guid. Contr. Dyn. 14, 304311.

Bohannan, G. [2002] Analog Realization of a Fractional Control Element - Revisited, pp. 175-182.

Butzer, P. L. \& Westphal, U. [2000] "An introduction to fractional calculus," Applications of Fractional Calculus in Physics, ed. Hilfer, R. (World Scientific, Singapore), pp. 1-85.
Caputo, M. [1967] "Linear models of dissipation whose $\mathrm{Q}$ is almost frequency independent-II," Geophys. J. Roy. Astron. Soc. 13, 529-539.

Chua, L. O. [1971] "Memristor-the missing circuit element," IEEE Trans. Circuit Th. 18, 507-519.

Chua, L. O. \& Kang, S. M. [1976] "Memristive devices and systems," Proc. IEEE 64, 209-223.

Chua, L. O. [2003] "Nonlinear circuit foundations for nanodevices, Part I: The four-element torus," Proc. IEEE 91, 1830-1859.

Chua, L. O. [2009] Introduction to Memristors, IEEE Expert Now, Educational Course.

Chua, L. O. [2011] "Resistance switching memories are memristors," Appl. Phys. A: Mater. Sci. Process. 102, $765-783$.

Chua, L. O. [2012] "The fourth element," Proc. IEEE 100, 1920-1927.

Coopmans, C., Petráš, I. \& Chen, Y. Q. [2009] "Analogue fractional-order generalized memristive devices," Proc. ASME 2009. Int. Design Engineering Technical Conf., Computers and Information in Engineering, pp. 1-10.

Di Ventra, M., Pershin, Y. V. \& Chua, L. O. [2009] "Circuit elements with memory: Memristors, memcapacitors, and meminductors," Proc. IEEE 97, 1717-1724.

Gale, E., Mayne, R., Adamatzky, A. \& de Lacy Costello, B. [2014] "Drop-coated titanium dioxide memristors," Mater. Chem. Phys. 143, 524-529.

Heaviside, O. [1971] Electromagnetic Theory (Chelsea, $\mathrm{NY}$ ).

Johnsen, G. K. [2012] "An introduction to memristor - A valuable circuit element in bioelectricty and bioimpedance," J. Electr. Bioimped. 3, 20-28.

Kusnezov, D., Bulgac, A. \& Dang, G. D. [1999] "Quantum levy processes and fractional kinetics," Phys. Rev. Lett. 82, 1136-1139.

Leibniz, G. W. [1962] Leibnizens Mathematische Schriften (Georg Ohms Verlagsbuch Handlung Hildesheim).

Le MeHaute, A. \& Crepy, G. [1983] "Introduction to transfer and motion in fractal media: The geometry of kinetics," Solid State Ionics 9-10, 17-30.

Podlubny, I. [1999] Fractional Differential Equations (Academic Press, San Diego).

Radwan, A. G., Soliman, A. M. \& Elwakil, A. S. [2008] "First-order filters generalized to the fractional domain," J. Circuits Syst. Comput. 17, 55-66.

Samko, S. G., Kilbas, A. A. \& Marichev, O. I. [1993] Fractional Integrals and Derivatives: Theory and Applications (Gordan and Breach, Amsterdam).

Strukov, D. B., Snider, G. S., Stewart, D. R. \& Williams, R. S. [2008] "The missing memristor found," Nature 453, 80-83.

Sun, H. H., Abdelwahab, A. A. \& Onaral, B. [1984] "Linear approximation of transfer function with a pole 
of fractional order," IEEE Trans. Autom. Contr. 29, 441-444.

Szabo, J. \& Abonyi, I. [1965] "Generalized Ohm's law in a magnetic plasma," Beiträge aus der Plasmaphysik 5, 9-12.
Tavazoei, M. S. [2010] "A note on fractional-order derivatives of periodic functions," Automatica 46, 945948. 